\title{
Analysis of the Wind Field Evolution Associated with the Extratropical Transition of Bonnie (1998)
}

\author{
Clark Evans and Robert E. Hart \\ Department of Meteorology, The Florida State University, Tallahassee, Florida
}

(Manuscript received 10 October 2006, in final form 24 August 2007)

\begin{abstract}
Extratropical transition brings about a number of environmentally induced structural changes within a transitioning tropical cyclone. Of particular interest among these changes is the acceleration of the wind field away from the cyclone's center of circulation along with the outward movement of the radial wind maximum, together termed wind field expansion. Previous informal hypotheses aimed at understanding this evolution do not entirely capture the observed expansion, while a review of the literature shows no formal work done upon the topic beyond analyzing its occurrence. This study seeks to analyze the physical and dynamical mechanisms behind the wind field expansion using model simulations of a representative transition case, North Atlantic Tropical Cyclone Bonnie of 1998. The acceleration of the wind field along the outer periphery of the cyclone is found to be a function of the net import of absolute angular momentum within the cyclone's environment along inflowing trajectories. This evolution is shown to be a natural outgrowth of the development of isentropic conveyor belts and asymmetries associated with extratropical cyclones. Asymmetries in the outer-core wind field manifest themselves via the tightening and development of height and temperature gradients within the cyclone's environment. Outward movement of the radial wind maximum occurs coincident with integrated net cooling found inside the radius of maximum winds. Tests using a secondary circulation balance model show the radial wind maximum evolution to be similar yet opposite to the response noted for intensifying tropical cyclones with contracting eyewalls.
\end{abstract}

\section{Introduction}

The extratropical transition (ET) of tropical cyclones (TCs) is the process by which a TC transforms into an extratropical (or midlatitude) cyclone. An ET is brought about by the interaction of the TC with the midlatitude baroclinic environment, an environment characterized by increased baroclinicity and vertical wind shear; meridional moisture gradients; decreasing sea surface temperatures (SSTs) and/or strong SST gradients; and increasing Coriolis force (Jones et al. 2003). This interaction results in an array of structural changes within the cyclone, including the development of frontal structures, particularly warm frontal structures (Harr and Elsberry 2000); enhanced wave heights to the right of the cyclone's motion (Bowyer 2000); and asymmetric evolutions of the cloud, precipitation, and wind fields (Jones et al. 2003, and references therein).

Corresponding author address: Clark Evans, 404 Love Building (MC 4520), 1017 Academic Way, Tallahassee, FL 32306-4520.

E-mail: acevans@met.fsu.edu
The conceptual model of Klein et al. (2000) provides a concise depiction of these structural changes from an observational standpoint, while the cyclone phase space of Hart (2003) provides a means for analyzing and predicting such structural changes (Evans and Hart 2003). Beyond a purely meteorological standpoint, ET is a topic of particular interest to society given that its impacts can be even more costly and deadly than a purely tropical or a purely extratropical cyclone and affect regions such as Japan, New England, and the Canadian Maritimesregions that typically do not see such strong cyclones except during the cold season (Jones et al. 2003).

Of particular interest is the wind field expansion associated with ET events. This wind field expansion can be characterized by two components: the outward movement of the radius of maximum winds (RMW; Jones et al. 2003) and a flattening of the azimuthally averaged tangential wind profile concordant with an acceleration of the wind field outside the RMW, as will be shown later. Little research has been performed towards understanding this phenomenon, with most attention paid to its occurrence (e.g., the model analyses

DOI: 10.1175/2007MWR2051.1 
of Ritchie and Elsberry 2001) rather than to its underlying causes. Because of this limited understanding, operational forecasts of the wind field evolution associated with transitioning TCs in the North Atlantic basin are often formulated with a relatively large focus upon climatology, persistence, and structural features shown in the various observation platforms (J. Beven 2006, personal communication). Further, informal hypotheses proposed to explain their evolution, including an increase of the Coriolis force and the movement of air parcels outward from the cyclone's center, do not fully account for the observed evolution during ET (Evans 2006). The former theory fails because of the conservation of absolute vorticity, whereby for increasing Coriolis force one expects decreasing relative vorticity and ultimately decreased wind speeds; instead, absolute vorticity is not conserved as divergence in the midlatitudes is typically large. Furthermore, zonally moving cyclones (where the Coriolis force remains unchanged) also experience an expansion. The latter theory fails because of conservation of angular momentum as compared with the typical structure of a vortex under conditions of gradient wind balance. Thus, a disconnect exists between observing that the wind field expansion occurs and understanding why it does so.

The aim of this paper is to use a physical and dynamical perspective to understand the wind field expansion as a function of features influencing the ET process as a whole. Insight from tropical and extratropical cyclone development and evolution theories is utilized where appropriate to accentuate the expansion evolution. Details behind the analysis methodology, data, and techniques are presented in section 2. Results of the experiments and simulations performed are presented in section 3, with an extension to the findings explored using a secondary circulation nonlinear balance model in section 4. Implications and extensions of the findings are presented along with a concluding discussion in section 5 .

\section{Data and methodology}

\section{a. Case selection}

To perform experiments to better understand the wind field evolution during ET, a representative case of ET in the North Atlantic Ocean basin is selected-TC Bonnie of 1998 (Avila 1998). TC Bonnie provides an ideal case for analysis owing to several desired parameters: a cold-core ET event followed by the ultimate decay of the cyclone, with no "warm seclusion" (Shapiro and Keyser 1990) posttransition evolution or reintensification after ET; an identifiable wind field expansion in both operational model analyses and National
Hurricane Center (NHC) advisories (National Hurricane Center 2007); and no decay or merger with another cyclone at the completion of ET (Fig. 1 in Avila 1998). The TC Bonnie is deemed a representative coldcore ET case given the good agreement between its path through the cyclone phase space of Hart (2003; see also Fig. 1a) when compared with the composite evolution depicted in Hart et al. (2006, their Fig. 3a). Note that cold-core ET events account for approximately $70 \%$ of North Atlantic ET events between 1998 and 2003 (Hart et al. 2006), making understanding the wind field evolution associated with such transition events crucial to understanding its overall evolution during all ET events.

\section{b. Analysis methodology}

A simulation-based methodology is employed to study the evolution of TC Bonnie. This approach is employed in lieu of idealized modeling and real-data analysis approaches given the lack of a high-resolution four-dimensional dataset encompassing a transitioning cyclone and its environment and the ability of a model simulation to provide a means of verification and comparison with reality. Simulations are performed using the nonhydrostatic fifth-generation Pennsylvania State University-National Center for Atmospheric Research (NCAR) Mesoscale Model (MM5, version 3.7.2; Dudhia 1993) at $36 \mathrm{~km}$ and $12 \mathrm{~km}$ horizontal grid spacing. The $36-\mathrm{km}$ simulation is a 5-day simulation performed between the times 1200 UTC 26 August 1998 and 1200 UTC 31 August 1998, or from near the time of peak intensity through one day after the completion of ET [as determined by NHC advisories and the cyclone phase space of Hart (2003); see Fig. 1a]. Navy Operational Global Atmospheric Prediction System (NOGAPS) $1^{\circ}$ operational analyses supply the initial and boundary conditions for the $36-\mathrm{km}$ model simulation; note that these analyses include a synthetic vortex for the TC and that no attempt is made to remove this vortex or specify another vortex in its stead. The $12-\mathrm{km}$ simulation is performed as a one-way nest from the 36-km simulation data and is a 3-day simulation encompassing the time period between 1200 UTC 28 August 1998 and 1200 UTC 31 August 1998, or about one day prior to the start of ET through one day after the completion of ET. A full listing of relevant model parameters may be found in Table 1, while a graphical depiction of the $12-\mathrm{km}$ simulation domain can be found in Fig. 2a. Unless otherwise noted, all results presented are based upon output from the $12-\mathrm{km}$ simulation and are specific to the simulated atmosphere.

Subjective and objective analyses of the model output compared with both observations and available op- 
(a)

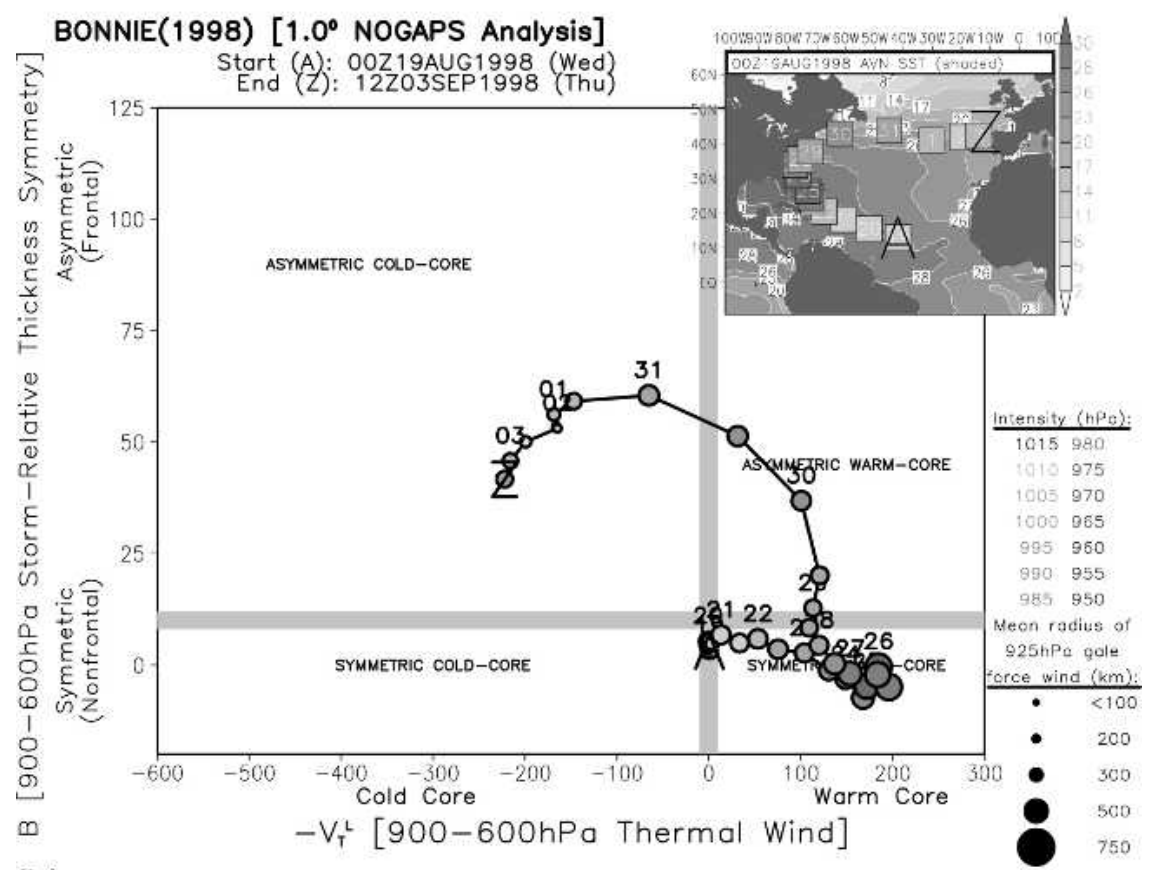

(b)

BONNIE(1998) [12km MM5 Analysis]

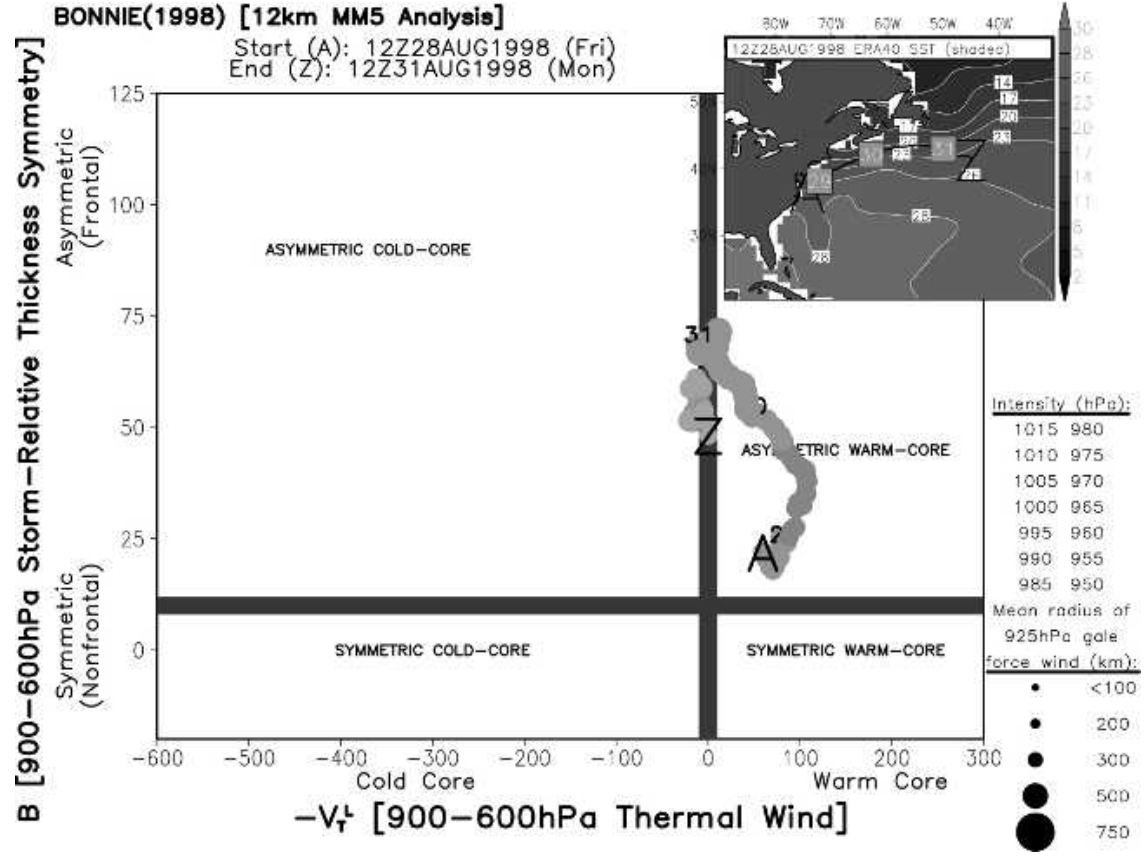

FIG. 1. (a) The 900-600-hPa thickness vs 900-600-hPa-layer thermal wind phase space diagram during the life cycle of TC Bonnie from $1^{\circ}$ NOGAPS analyses; (b) as in (a), but from the 12-km MM5 simulation output between 1200 UTC 28 Aug and 1200 UTC 31 Aug 1998. For a complete description of the parameters detailed here, see Hart (2003).

erational analysis products show that the model simulations capture the evolution of the transitioning cyclone and its environment reasonably well. The simulated intensity is slightly stronger than analyzed in the postseason cyclone analysis (Figs. 2b,c), potentially due to the initial synthetic vortex found within the NOGAPS analyses (e.g., Evans et al. 2006). Furthermore, the initial surface wind field from the $12-\mathrm{km}$ 
TABLE 1. Model parameters selected for use with the 36- and 12-km MM5 simulations of TC Bonnie.

\begin{tabular}{lll}
\hline \multicolumn{1}{c}{ Model parameter } & \multicolumn{1}{c}{$36-\mathrm{km}$ simulation } & \multicolumn{1}{c}{$12-\mathrm{km}$ simulation } \\
\hline Size of model domain (model grid points) & $250 \times 300 \times 30$ & $301 \times 301 \times 30$ \\
Center of domain & $40^{\circ} \mathrm{N}, 60^{\circ} \mathrm{W}$ & Approx $38^{\circ} \mathrm{N}, 65^{\circ} \mathrm{W}$ \\
Top of model & $50 \mathrm{hPa}$ & $50 \mathrm{hPa}$ \\
Analysis period & $1200 \mathrm{UTC} 26$ Aug-1200 UTC & $1200 \mathrm{UTC} 28$ Aug-1200 UTC \\
& 31 Aug 1998 & 31 Aug 1998 \\
Initial/boundary conditions & NOGAPS $1^{\circ}$ analyses & MM5 36-km simulation output \\
Forecast length & $120 \mathrm{~h}$ & $72 \mathrm{~h}$ \\
Forecast output interval & $3 \mathrm{~h}$ & $1 \mathrm{~h}$ \\
Moisture scheme & Mixed phase (Reisner et al. 1998) & Mixed phase (Reisner et al. 1998) \\
Cumulus parameterization & Kain-Fritsch (Kain and Fritsch 1993) & Kain-Fritsch (Kain and Fritsch 1993) \\
Boundary layer scheme & Blackadar (Blackadar 1979) & Blackadar (Blackadar 1979) \\
\hline
\end{tabular}

MM5 simulation compares reasonably well to observations (Fig. 3) in terms of structure, although maximum winds are significantly weaker. Observations $3 \mathrm{~h}$ later (not shown), however, show a much weaker cyclone, bringing the simulated and observed wind fields into better agreement. While the precise intensity is not well captured by the model simulation, particularly in its first $24 \mathrm{~h}$, it is argued that there is sufficient agreement with respect to the overall evolution of the cyclone during ET to not substantially impact the analyses presented in this work.

Simulation cyclone track errors are on the order of the NHC long-term 3-5-day North Atlantic forecast track errors (National Hurricane Center 2006). Differences in the simulated tracks from reality arise because of slower forward motion in the simulations, where the deep-layer midlatitude steering flow is slower in accelerating the storm eastward (not shown). However, this does not result in any significant structural differences as gauged by the thermal and dynamical structure of the cyclone (cf. Figs. 1a,b). This arises because the midlatitude trough of low pressure influencing ET was of similar intensity, orientation, and distance away from the cyclone in the simulations as in reality. Thus, it is further argued that the model simulation data are of good quality, though it is acknowledged that every possible detail may not be accounted for within the simulation results and analyses.

Analyses using a combination of azimuthally averaged and nonazimuthally averaged fields are employed to study the synoptic-scale and mesoscale evolutions, respectively, of the cyclone's wind field. Azimuthally averaged fields have the benefit of accounting for any impact of storm motion that may appear within the wind field, making the observed wind field evolution storm-motion independent. Further, they inherently incorporate the contributions of wind field asymmetries to the broader-scale wind field evolution. Nonazimuthally averaged fields are used to supplement the azi- muthally averaged analyses where appropriate to study mesoscale features that contribute to the larger-scale wind field evolution. It is this blend of analysis methods that allows us to both capture the overall wind field evolution as well as study its contributors in some detail.

\section{Results}

\section{a. Evidence of expansion}

A clear evolution of the wind field of TC Bonnie is noted throughout the simulation period and thus, by extension, the ET process, with respect to both the outward movement of the RMW and the acceleration of the wind field at outer radii. Figure 4 a depicts the evolution of the $10-\mathrm{m}$ azimuthally averaged wind field through the duration of the $12-\mathrm{km}$ simulation period, including the entire ET time period. An outward displacement of the RMW from approximately $100 \mathrm{~km}$ to approximately $200 \mathrm{~km}$ is noted in conjunction with an acceleration of the wind field at outer radii (greater than $300-\mathrm{km}$ radius) of approximately $4 \mathrm{~m} \mathrm{~s}^{-1}$ over a 36-48-h time frame. The greatest wind field acceleration and outward displacement occur after 0000 UTC 30 August 1998, approximately $12 \mathrm{~h}$ prior to the completion of ET. A similar evolution to that at $10 \mathrm{~m}$ is noted on the 310-K (approximately 600-700 hPa; Fig. 4b) and 324-K (approximately 500-600 hPa; Fig. 4c) isentropic surfaces, with the onset of the expansion process occurring at approximately the same time but with a faster and more significant outer wind field acceleration and flattening of the cyclone's tangential wind profile. To better depict the observed wind field evolution, slices through the 10 -m time-radius profile at three selected times-0400 UTC 29 August 1998 (before ET onset), 1000 UTC 30 August 1998 (near ET completion), and 1000 UTC 31 August 1998 (after ET completion)-are presented in Fig. 5. The time evolution of the lower-level wind field clearly shows the joint evolution of the two wind field expansion components, 
(a)

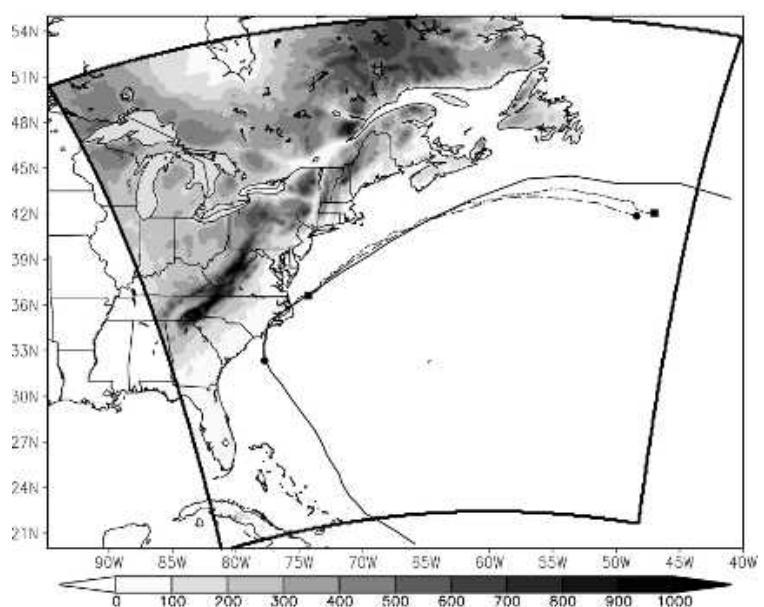

(b)

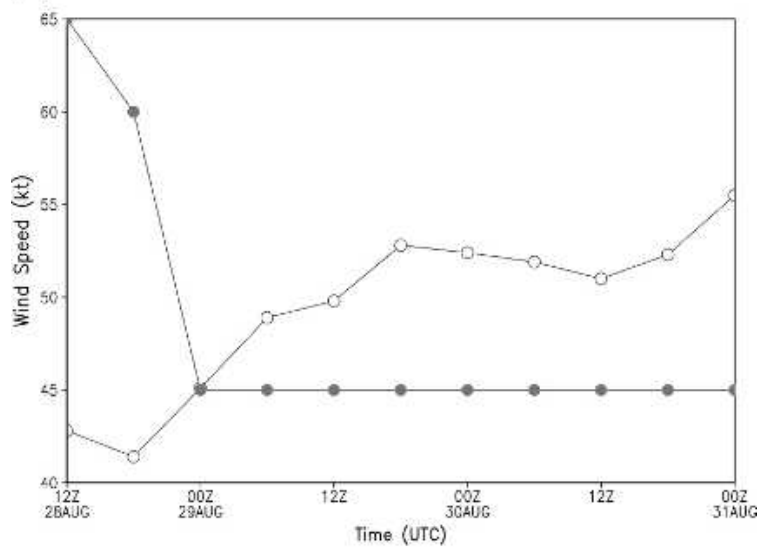

(c)

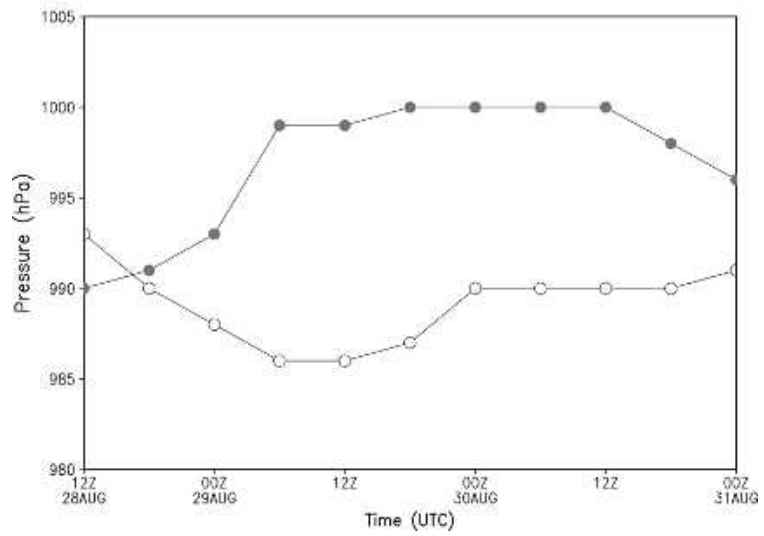

FIG. 2. (a) Graphical depiction of the 12-km MM5 simulation domain (terrain elevation shaded; $\mathrm{m}$ ) and comparison of the NHC best track (solid), 36-km MM5 simulation (long dash), and 12-km MM5 simulation (short dash) tracks of TC Bonnie. The ending time for all three is 0600 UTC 31 Aug 1998, the end of the NHC best track. (b) The 12-km MM5 (open circles) and NHC best track (closed circles) maximum 10-m wind speed (kt) between 1200 UTC 28 Aug and 0000 UTC 31 Aug 1998. (c) As in (b), but for the minimum sea level pressure $(\mathrm{hPa})$.

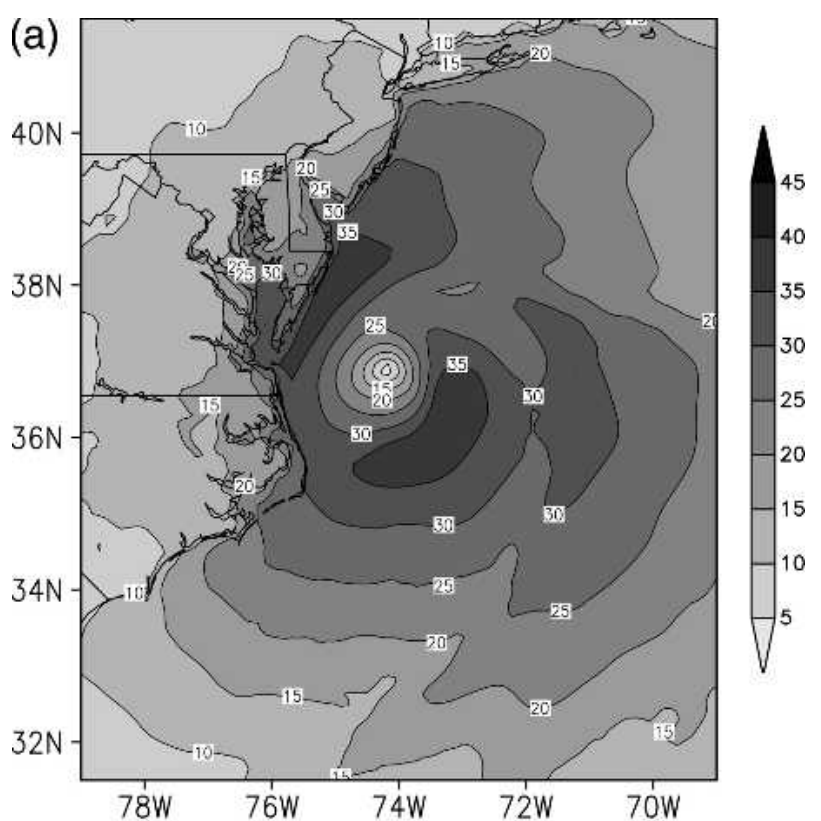

(b)

ATTENTION: HURRICANE SPECIALISTS

Hurricane Bonnie 1330 UTC 28 August 1998

Max. 1-min sustained surface winds (kt) for marine exposure Analissis based on AFRC C- 130 Recon cata from $700 \mathrm{mb}$ STC adjusted: $0930-1245$; MAN, buoys, ships from $0900-12002$

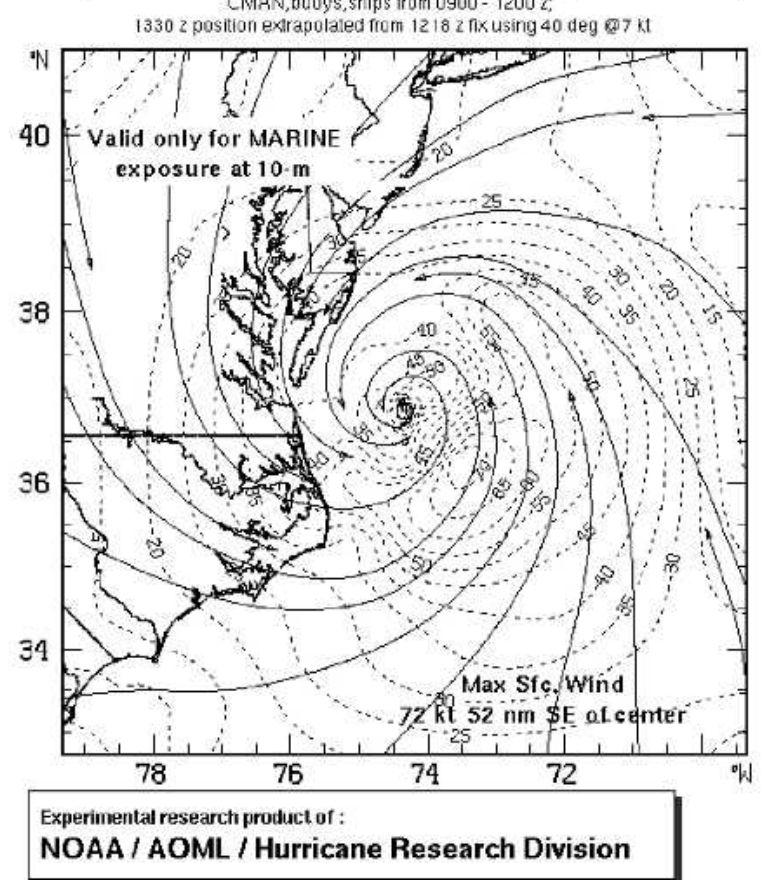

Fig. 3. (a) Maximum 10-m wind field (kt) at 1300 UTC 28 Aug 1998 from 12-km MM5 simulation output; (b) as in (a), but at 1330 UTC 28 Aug 1998 from the Hurricane Research Division (Powell et al. 1998; available online at http://www.aoml.noaa.gov/hrd/ Storm_pages/bonnie1998/wind.html). 


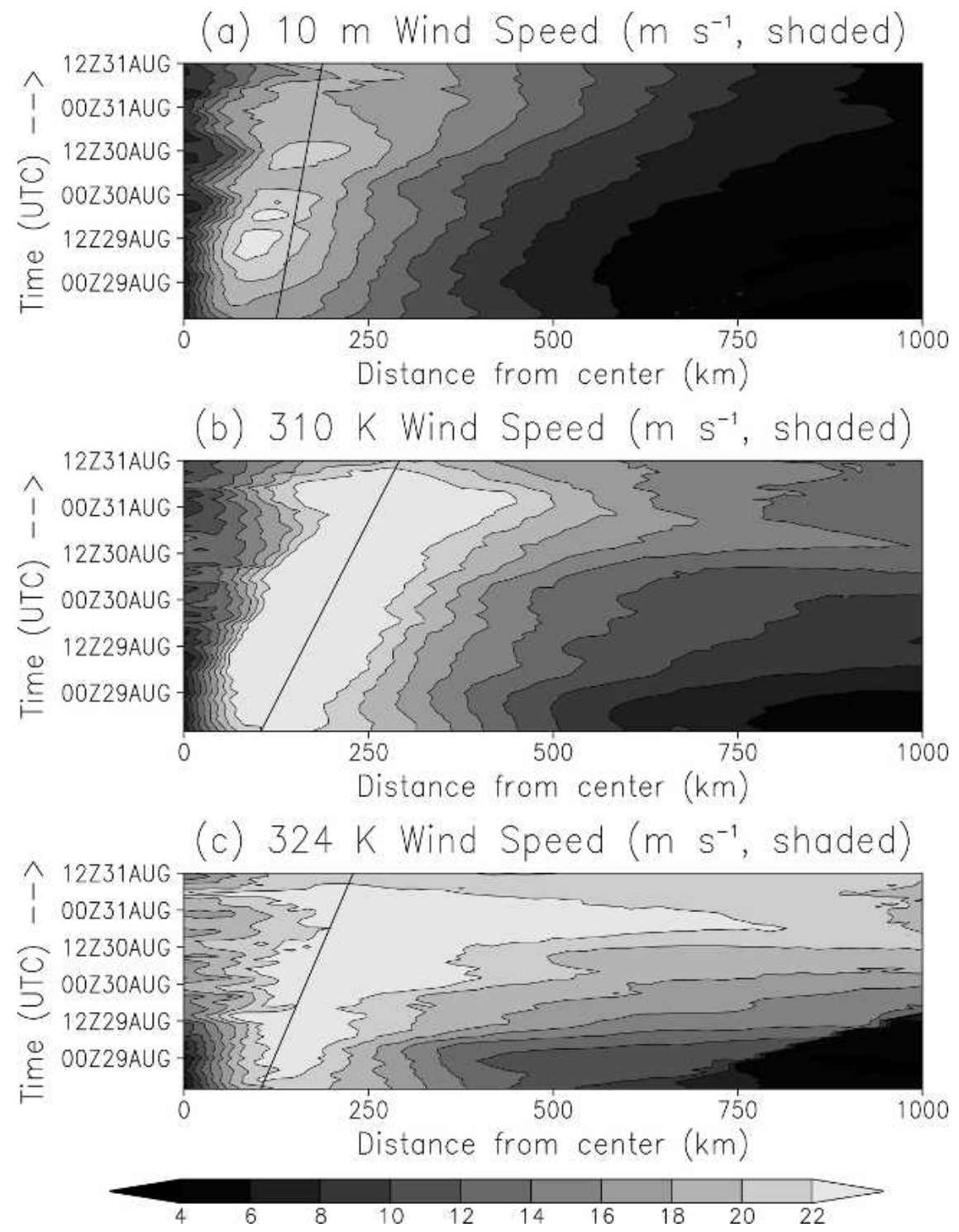

Fig. 4. (a) Azimuthally averaged $10-\mathrm{m}$ wind field $\left(\mathrm{m} \mathrm{s}^{-1}\right)$ between 0 - and 1000-km radius during the period from 1200 UTC 28 Aug to 1200 UTC 31 Aug 1998; (b) as in (a), but on the $310-\mathrm{K}$ isentropic surface; (c) as in (a), but on the $324-\mathrm{K}$ isentropic surface. The radii of maximum winds at the start and end of the $12-\mathrm{km}$ model simulation are connected in (a)-(c) by the solid black line.

with a gradual acceleration of the outer wind profile and outward movement of the RMW throughout time.

In all, these evolutions suggest that the wind field expansion in Bonnie is a top-down process, occurring most significantly first at upper-tropospheric levels and later at lower-tropospheric levels. This fits naturally with the evolution of the cyclone's thermodynamic structure during ET in association with a midlatitude trough of low pressure, with the upper-level warm core eroding first near the start and the lower-level warm core doing the same near the end of ET (Evans and
Hart 2003). Further evidence of this evolution is provided by isentropic radial cross sections through the cyclone (Fig. 6) at five times during the model simulation, three of which are the same times as presented in Fig. 5. Expansion at outer radii first occurs as the cyclone begins to interact with the midlatitude environment at upper levels, progressively expanding at lower levels as time wears on.

Significant asymmetries are found within this largerscale azimuthally averaged wind field evolution, as shown in Fig. 7. At 10 m (Figs. 7a,b), an expansion of 


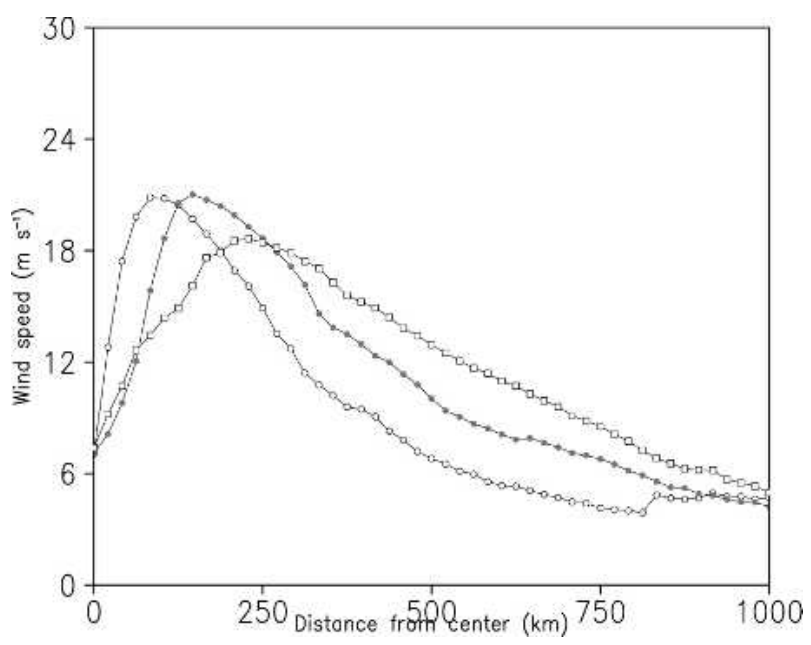

FIG. 5. Time slices through the time-radius $10-\mathrm{m}$ wind profile $\left(\mathrm{m} \mathrm{s}^{-1}\right)$ presented in Fig. 3 at 0400 UTC 29 Aug (open circles; before ET), 1000 UTC 30 Aug (closed circles; during ET), and 1000 UTC 31 Aug (open squares; after ET) 1998.

the storm-force (16-20 $\mathrm{m} \mathrm{s}^{-1}$ ) wind radii is noted in all quadrants during ET, with the most significant expansion (or acceleration) noted in the northwest quadrant-associated with a narrow wind maximum - and right of the cyclone's motion. A similar evolution is noted on the 310-K (Figs. 7c,d) and 324-K (Figs. 7e,f) isentropic surfaces, particularly in terms of the right of track expansion as associated with the development of a low-level jet stream. A relative minimum is present in the cyclone's northwest quadrant on the $310-\mathrm{K}$ surface (Fig. 7d), opposite to that on the 10-m surface (Fig. 7b). Note that these asymmetries are largely storm-motion independent (not shown) and that the overall wind field evolution is entirely storm-motion independent, as confirmed by the azimuthally averaged evolution previously shown (e.g., Fig. 6).

\section{b. Outer-core evolution}

\section{1) Primary circulation ANd airstream EVOLUTION}

In discussing the outer-core evolution of the transitioning cyclone's wind field, it is the acceleration of the wind field at radii outside the RMW that is under consideration, or the expansion outside $200 \mathrm{~km}$ in radius noted in Figs. 4-6. First, to understand the factors that influence the outer-core wind field evolution, it is critical to understand the more general evolution of the transitioning cyclone's airstreams and circulation itself. Nominally, as a cyclone undergoes ET, it becomes increasingly asymmetric (as highlighted by the cyclone phase space diagram in Fig. 1b), resulting in increased radial flow both into and out of the cyclone associated with its primary circulation. This follows from both the conceptual model of ET of Klein et al. (2000) and the development of conveyor belts such as those of Browning (1999). To attempt to quantify the development of these conveyor belts and better understand the cyclone's circulation changes, trajectory analyses are computed in space and time (Fig. 8a) and clustered into three general evolutions.

The first of these trajectory clusters is depicted by trajectories 1 and 4 in Fig. 8a. At and before ET (open circles in Fig. 8a), parcels associated with these trajectories rotate around the cyclone and rise to the east of the cyclone. During and near the end of ET (open squares in Fig. 8a), these parcels are recaptured by the cyclone in its developing cold conveyor belt, descend to lower altitudes, and ultimately are evacuated from the cyclone's environment by its warm conveyor belt and upper-level steering environment. Closely related to this trajectory cluster is a second cluster, representing trajectories 3, 5, and 6. Like the first cluster, these parcels start off south and east of the cyclone's center and rise in convective elements to its east. However, as ET progresses, these parcels are already well east of the cyclone at upper levels; they are not recaptured by the cyclone's developing cold conveyor belt. In fact, at the end of ET, all of these parcels are outside the model simulation domain. The final trajectory cluster, represented by a sole trajectory (trajectory 2), starts over the Great Lakes region and slowly descends into the cyclone via its developing dry intrusion. Parcels in this cluster, much like parcels in the other two trajectory clusters, are ultimately caught in the cyclone's developing warm conveyor belt and evacuated outside the cyclone's environment at upper levels. Each of these parcel trajectories closely resembles those highlighted by the composite analysis of Klein et al. (2000) and the conveyor belt model of Browning (1999). Supporting evidence for this assertion is provided for the dry intrusion by descending, inflowing trajectories advecting in lower values of equivalent potential temperature and lower mixing ratio values on the $310-\mathrm{K}$ isentropic surface (Fig. 8b). For the warm conveyor belt, supporting evidence is given by low-level ascent over a baroclinic zone with well-defined advection of higher values of equivalent potential temperature to the east and southeast of the cyclone in a $25-40 \mathrm{~m} \mathrm{~s}^{-1}$ low-level jet (Fig. 8b).

\section{2) Azimuthally averaged evolution}

Given the interaction of the TC with an external momentum source in the form of an upper-level trough of low pressure and the observed trajectory analysis, it 
(a)

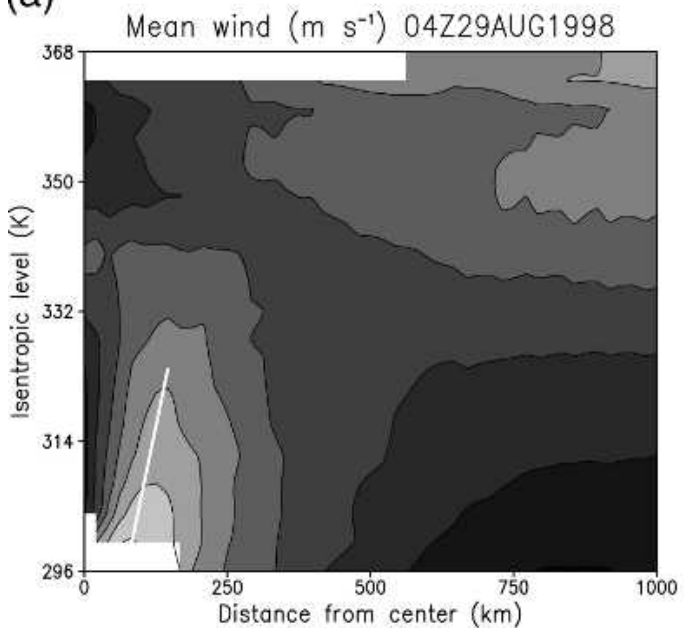

(c)

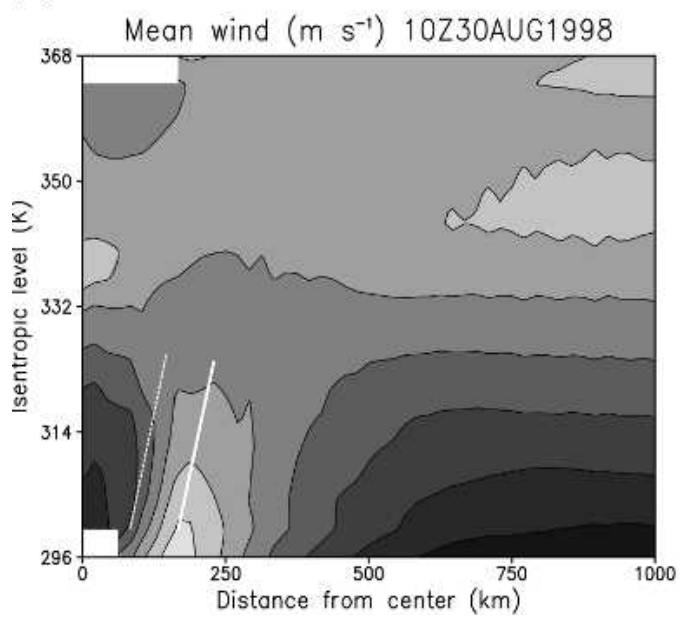

(e)

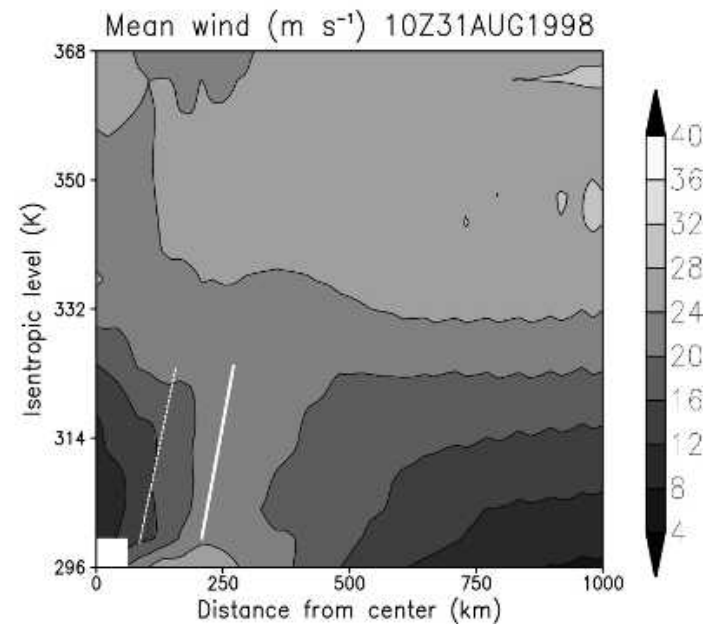

(b)

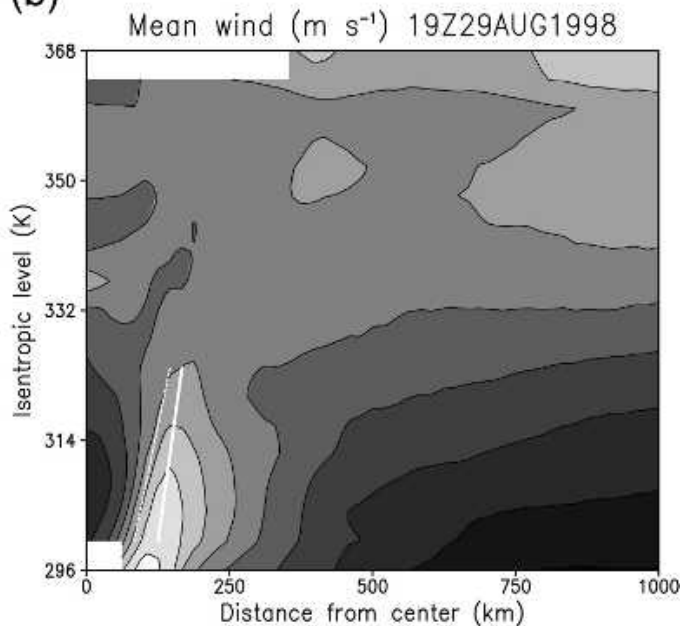

(d)

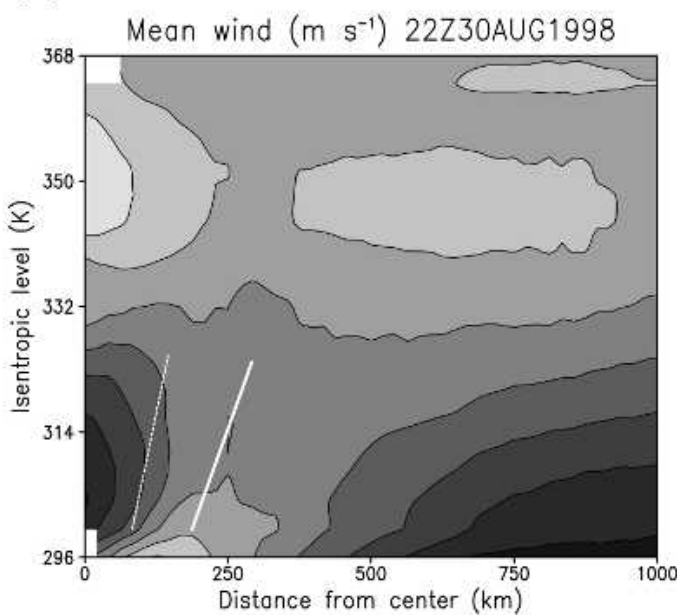

FIG. 6. Cross sections of the tangential wind field on isentropic surfaces between 296 and $368 \mathrm{~K}$ azimuthally averaged between 0 and $1000 \mathrm{~km}$ at (a) 0400 UTC 29 Aug, (b) 1900 UTC 29 Aug, (c) 1000 UTC 30 Aug, (d) 2200 UTC 30 Aug, and (e) 1000 UTC 31 Aug 1998. The radii of maximum winds at 300 and $320 \mathrm{~K}$ are connected in (a)-(e) by the solid white line; the line in (a) is duplicated in (b)-(e) by the dashed white line. 
(a) $10 \mathrm{~m}$ wind $\left(\mathrm{m} \mathrm{s}^{-1}\right)$ 04Z29AUG1998

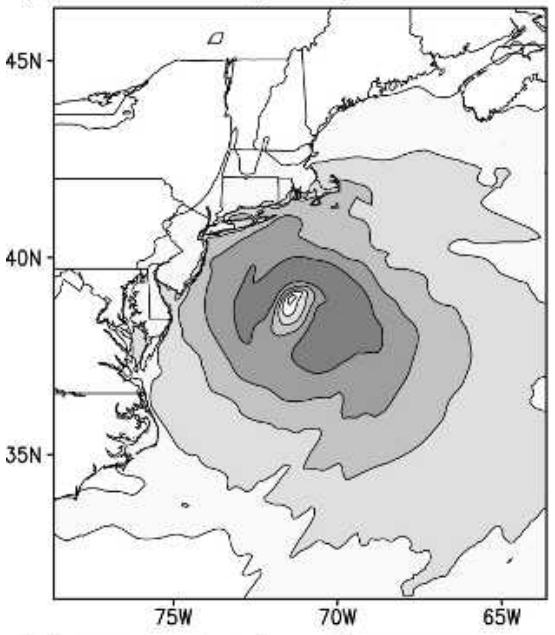

(b) $310 \mathrm{~K}$ wind $\left(\mathrm{m} \mathrm{s}^{-1}\right)$ 04Z29AUG1998

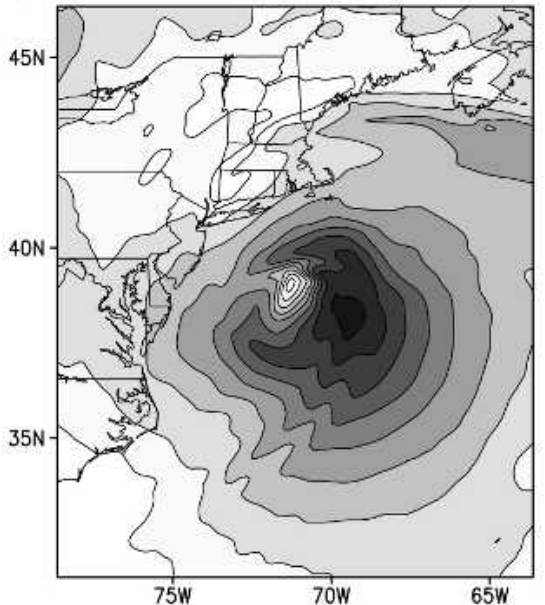

(c) $324 \mathrm{~K}$ wind $\left(\mathrm{m} \mathrm{s}^{-1}\right)$ 04Z29AUG1998

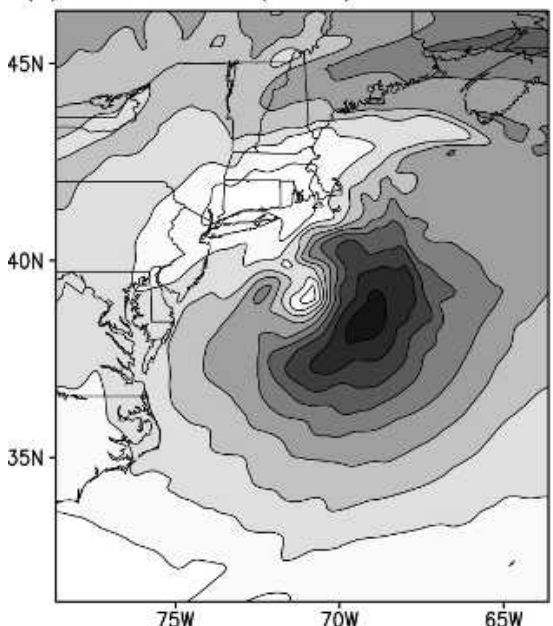

(d) $10 \mathrm{~m}$ wind $\left(\mathrm{m} \mathrm{s}^{-1}\right)$ 10Z30AUG1998
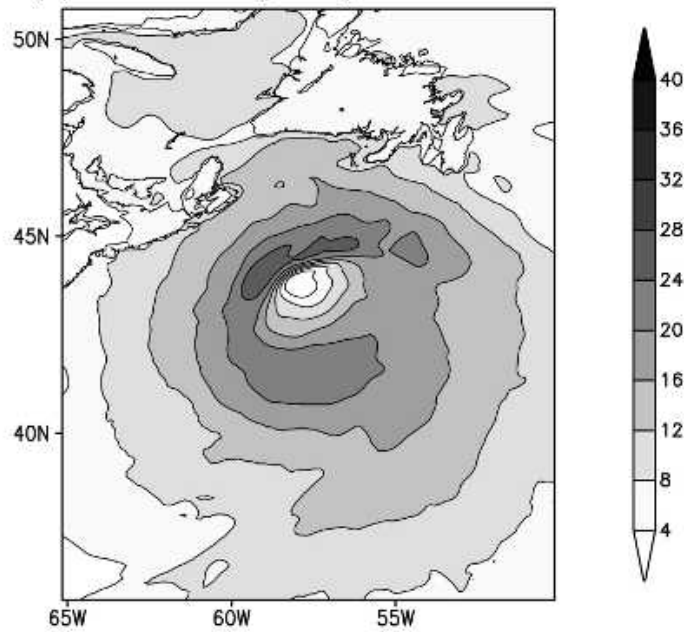

(e) $310 \mathrm{~K}$ wind $\left(\mathrm{m} \mathrm{s}^{-1}\right)$ 10Z30AUG1998

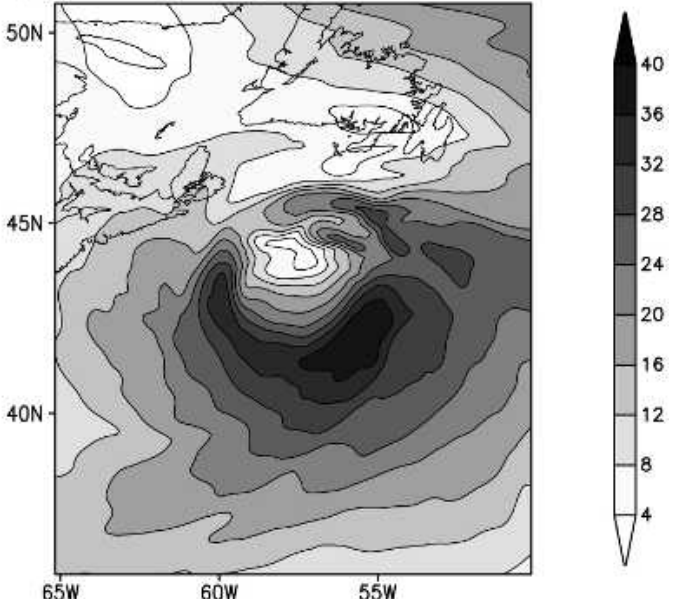

(f) $324 \mathrm{~K}$ wind $\left(\mathrm{m} \mathrm{s}^{-1}\right)$ 10Z30AUG1998

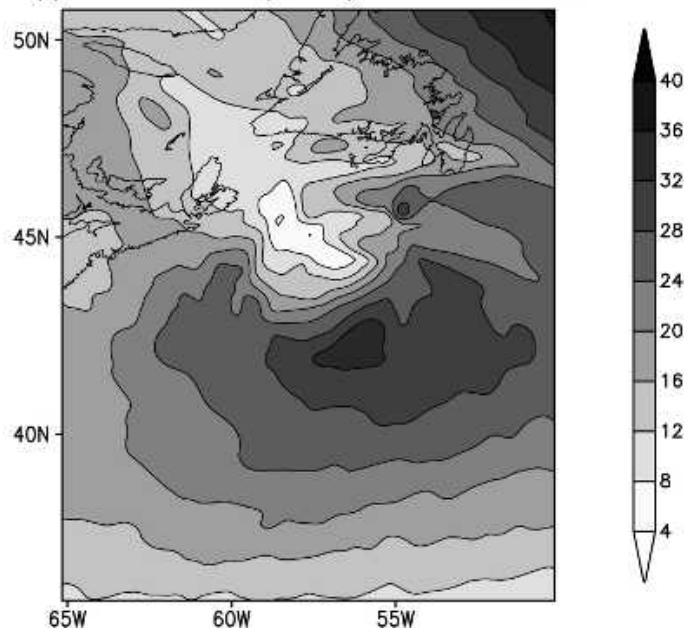

FIG. 7. Wind field $\left(\mathrm{m} \mathrm{s}^{-1}\right)$ analyses at 0400 UTC 29 Aug 1998 on the (a) 10-m surface, (c) 310-K isentropic surface, and (e) 324-K isentropic surface. (b), (d), and (f) As in (a), (c), and (e), respectively, but at 1000 UTC 30 Aug 1998. All analyses are over a domain of $15^{\circ} \times 15^{\circ}$ centered on the center of the cyclone at each time. 
(a)

TC Bonnie $12 \mathrm{~km}$ MM5 Trajectories (ribbons)

LEGEND

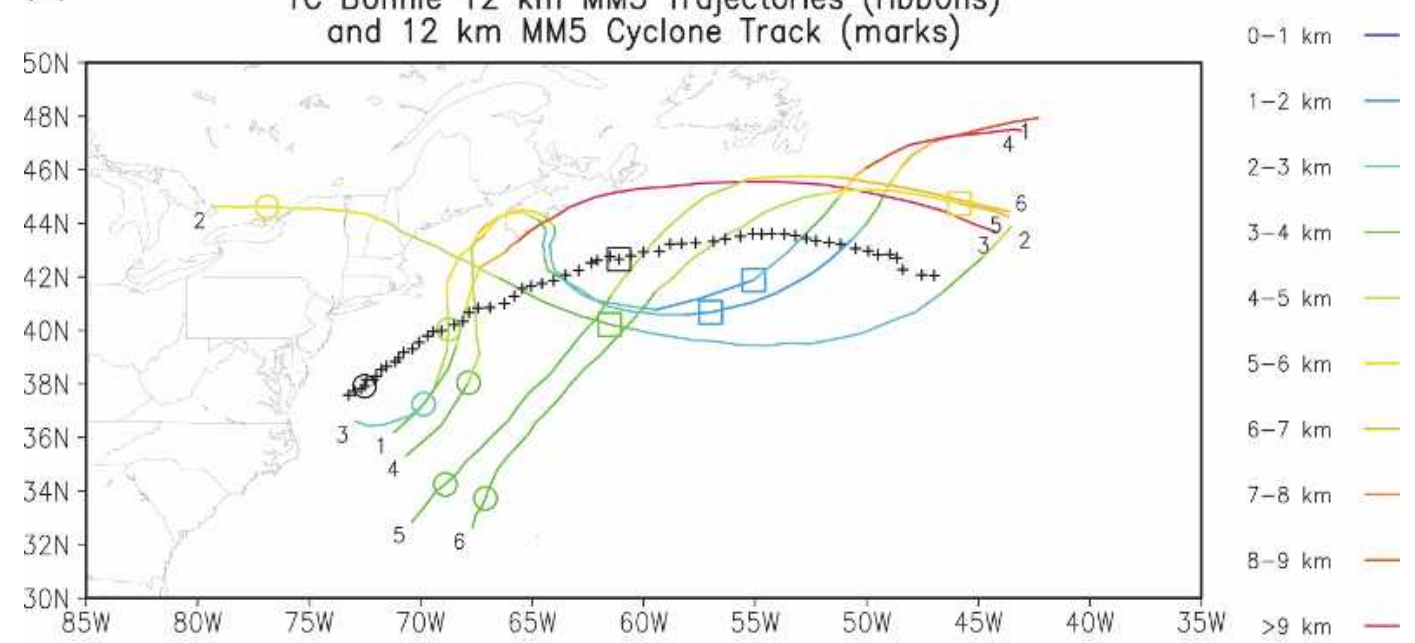

(b)

$310 \mathrm{~K}$ theta-e advection $\left(10^{-3} \mathrm{~K} \mathrm{~s}^{-1}\right.$, shaded)

mixing ratio $\left(\mathrm{g} \mathrm{kg}^{-1}\right.$, contour) and winds $\left(\mathrm{m} \mathrm{s}^{-1}\right.$, vector)

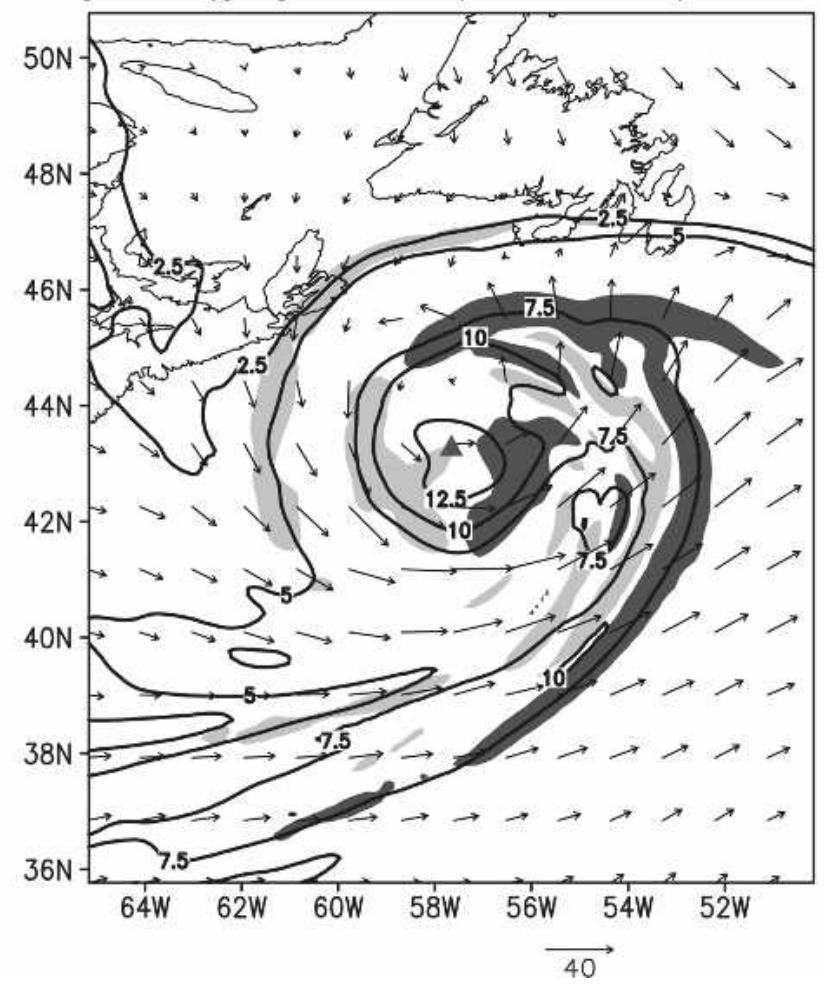

FIG. 8. (a) Selected trajectories about the circulation and environment of TC Bonnie (ribbons) and 12-km MM5 simulated track of TC Bonnie (marks) between 0000 UTC 29 Aug and 1200 UTC 31 Aug 1998. Color shading depicts the altitude $(\mathrm{km})$ of the parcel, open circles depict parcel location at 0400 UTC 29 Aug 1998, and open squares depict parcel location at 1000 UTC 30 Aug 1998. (b) Equivalent potential temperature advection (K s ${ }^{-1}$, shaded), mixing ratio ( $\mathrm{kg} \mathrm{kg}^{-1}$, contour), and wind field $\left(\mathrm{m} \mathrm{s}^{-1}\right.$, vector) centered on the storm on the $310-\mathrm{K}$ isentropic surface at 1000 UTC 30 Aug 1998. 
TABLE 2. Magnitudes of the four components of the angular momentum budget of McBride (1981b) as applied to the 12-km MM5 simulation data. Units of all terms are $10^{4} \mathrm{~kg} \mathrm{~s}^{-2}$ with positive values denoting an increase in momentum.

\begin{tabular}{lcccr}
\hline \hline & $\begin{array}{c}\text { Time } \\
\text { change }\end{array}$ & Transport & $\begin{array}{c}\text { Coriolis } \\
\text { torque }\end{array}$ & $\begin{array}{c}\text { Frictional } \\
\text { torque }\end{array}$ \\
\hline Bonnie1 & 0.0 & 34.97 & -9.78 & -9.37 \\
Bonnie2 & 0.0 & 27.74 & -4.00 & -11.68 \\
Danielle & 0.0 & 13.03 & -2.89 & -7.07 \\
McBride D4 & 0.0 & 11.70 & -3.50 & -5.20 \\
\hline
\end{tabular}

follows that momentum transport into and out of the cyclone's circulation may be occurring during ET, leading to the observed outer wind field evolution. To attempt to quantify whether this is the case, the vertically integrated angular momentum budget analysis of McBride (1981b) is applied to the model simulation data. This budget is obtained from the equation

$$
\frac{\partial}{\partial t}(m)=-\boldsymbol{\nabla} \cdot \mathbf{V} m-R f V_{R}+R F_{T},
$$

where $m=R V_{t}, F_{t}$ is a frictional torque and is calculated as in McBride (1981b), and all other terms have their standard meteorological meanings. The first term is the time rate of change of angular momentum, the second term is a transport term, the third term is a Coriolis torque, and the fourth term is a frictional torque. This equation is applied along a $500-\mathrm{km}$ radius band from the center of the cyclone at all times.

Subtle differences in calculation method are noted between the budgets of McBride (1981b) and those presented here. The McBride (1981b) budget uses the radiosonde composite data as outlined in McBride (1981a) with a resolution of $1^{\circ}(\sim 60-100 \mathrm{~km})$ in the radial direction, $45^{\circ}$ in the azimuthal direction, and with 21 vertical levels between the surface and $50 \mathrm{mb}$. The budgets presented here are designed to take advantage of the higher-resolution MM5 model simulation data and are interpolated to a cylindrical grid with a resolution of $25 \mathrm{~km}$ in the radial, $30^{\circ}$ in the azimuthal, and with 91 vertical height levels between the surface and 9 $\mathrm{km}$. Temporal variability is accounted for in the budgets presented here using a 6-h time average of the budget fields; in the McBride (1981b) budgets, such temporal variability is inherently smoothed out in their radiosonde compositing process.

Table 2 depicts the results of the momentum budget analysis for three simulation data periods: near the start of ET between 1800 and 2300 UTC 29 August 1998 (Bonnie1), near the end of ET between 0700 and 1200 UTC 30 August 1998 (Bonnie2), and for a purely tropical model simulation case between 2200 UTC $30 \mathrm{Au}$ - gust 1998 and 0300 UTC 31 August 1998 (Danielle), the latter of which is presented as a comparison case to the D4 "Atlantic Hurricane" composite of McBride (1981b). Good agreement is noted between the Danielle composite and the D4 composite of McBride (1981b) with similar values to all four terms. Compared with these two cases, however, the two Bonnie cases exhibit much greater momentum transport that is only slightly compensated for by increased Coriolis and frictional torques. The large magnitudes of the transport term during Bonnie's transition phase suggests that increased radial flow-such as that highlighted by the trajectory analysis - is leading to a net import of angular momentum within the cyclone's circulation as the cyclone's outer wind field is accelerating at all analyzed levels (Fig. 4). Thus, this suggests that the azimuthally averaged evolution of the outer wind field during ET is a natural outgrowth of the increasingly asymmetric nature of the cyclone brought about by its interaction with the midlatitude environment and thus a natural outgrowth of the ET process as a whole.

\section{3) Asymmetric evolution}

While the momentum budget analysis provides an overview of the evolution of the outer wind field as a whole, it does not fully capture the spatial (asymmetric) wind field evolution. To account for this, the horizontal momentum equation on isentropic surfaces of Shapiro (1975) is applied. This equation is given by

$$
\frac{\partial}{\partial t}(\mathbf{v})+\mathbf{v} \cdot \nabla_{\theta} \mathbf{v}-f \mathbf{k} \times \mathbf{v}+\nabla_{\theta} \psi=0,
$$

where subscripts of $\theta$ denote application on isentropic surfaces, $\psi$ is the Montgomery streamfunction and is equal to $g z+c_{p} T$, and boldface type denotes vector quantities. The first term is the velocity time tendency, the second term is the advection of the wind field, the third term is a Coriolis term, and the fourth term is a streamfunction term. The magnitudes of each of the last three terms are directly computed while the time tendency term is calculated as their residual. Note that these fields are instantaneous snapshots of the cyclone. A storm-centered perspective is applied to the analyses, with cyclonically directed components denoting wind field accelerations per the convention on the first term in (1) and inward-directed components denoting asymmetric (radial) evolutions within the wind field.

Figure 9 displays the magnitudes of these fields on the 310-K isentropic surface before (Fig. 9a) and during (Fig. 9b) ET. Displayed in the four panels are the advection term (upper left), Coriolis term (upper right), streamfunction term (lower left), and time 
(a)
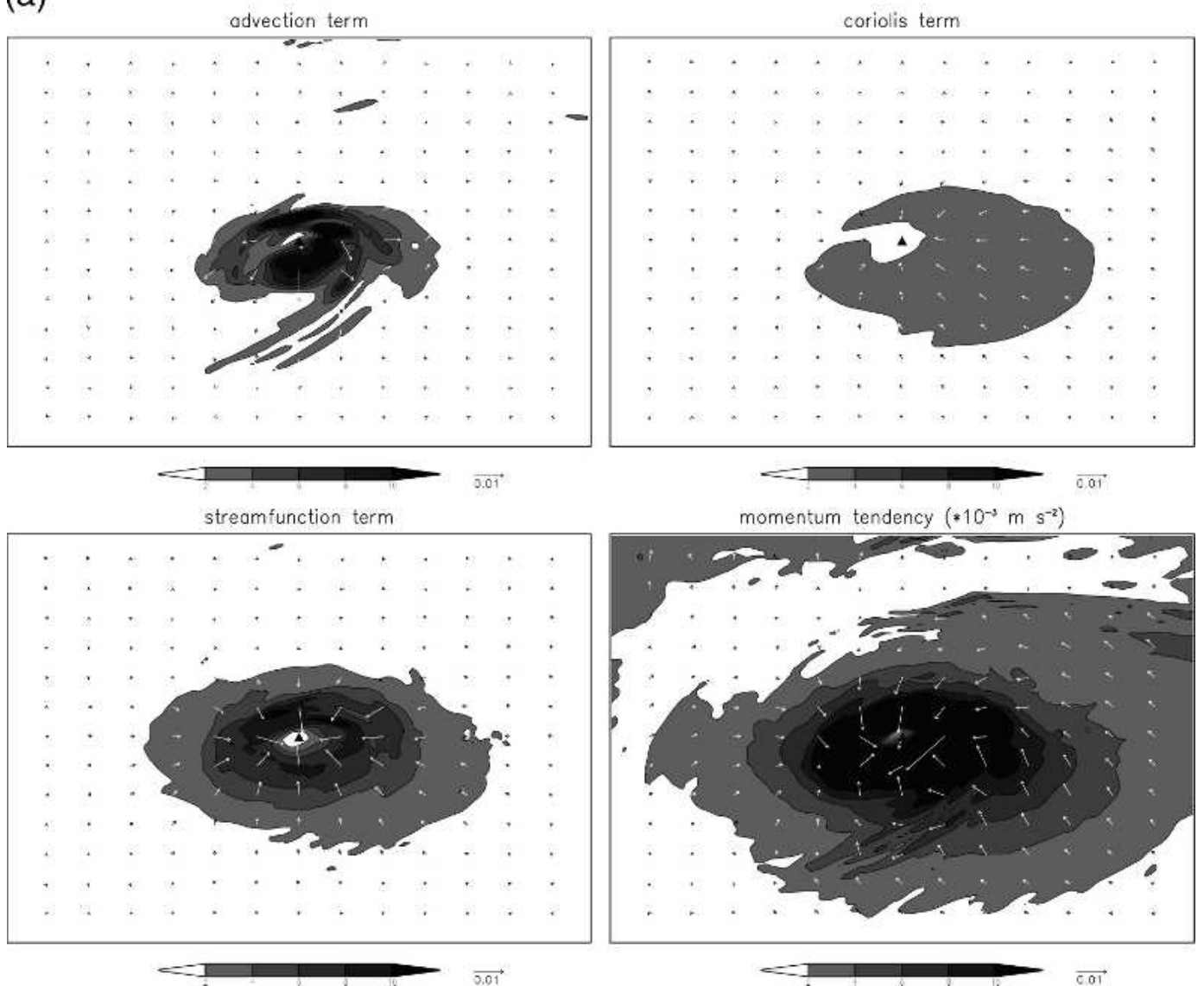

FIG. 9. Analyses of the isentropic momentum equation $\left(\mathrm{m} \mathrm{s}^{-2}\right)$ applied on the $310-\mathrm{K}$ isentropic surface at (a) 0400 UTC 29 Aug 1998 and (b) 1000 UTC 30 Aug 1998. Analyses are over a domain of $15^{\circ} \times 15^{\circ}$ centered on the center of the cyclone at each time, with the center depicted by the shaded triangle. Inward (outward)-pointing arrows denote acceleration (deceleration). Depicted are (upper left) the advection term, (upper right) Coriolis term, (lower left) streamfunction term, and (lower right) time tendency term.

tendency term (lower right). On isentropic surfaces, wind field changes occur primarily because of advective processes and the Montgomery streamfunction component to the isentropic momentum equation. This suggests that redistribution processes (by advection) and the evolution of height and temperature gradients - the two varying components to the Montgomery streamfunction-beyond the RMW are causing acceleration to the wind field. The response in the temperature and height gradients occurs at progressively outer radii through time on the $310-\mathrm{K}$ surface and is manifest through their tightening. Note that the regions of the strongest accelerations and most significant magnitudes of the advection and Montgomery streamfunction terms are found where trajectories are clustered during ET (Fig. 8a) and, expectedly, where the horizontal wind fields experience their greatest changes (cf. Fig. 9b with Figs. 7c,d). Furthermore, also note that the Coriolis component to the isentropic momentum equation is relatively small at all times. This implies that an increasing Coriolis parameter associated with ET cannot and does not solely account for the observed wind field evolution. The evolution of the terms of the isentropic momentum equation is consistent through time, suggesting that the factors responsible for the outer wind field evolution do not significantly change during ET.

Ultimately, the wind field accelerates in response to changes in the height and temperature gradient fields in order to maintain gradient wind balance as angular momentum is imported into the cyclone's outer circulation envelope. These height and temperature gradient changes are a natural outgrowth of the ET process, where the cyclone moves into an environment featuring significant temperature and height gradients (Jones et al. 2003) and its thermal structure changes from warm 
(b)

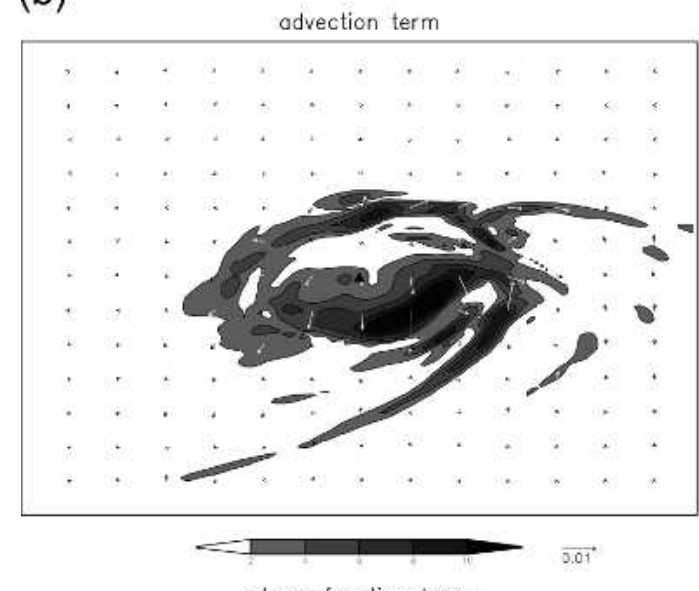

streamfunction term

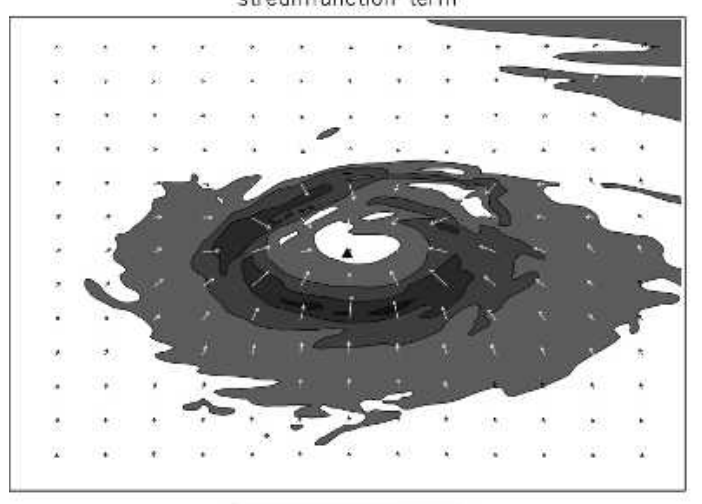

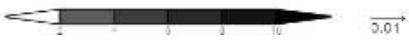

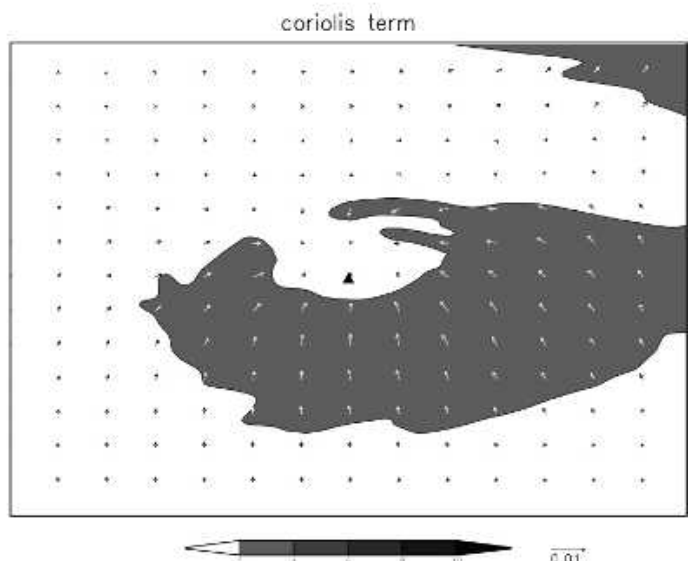

momentum tendency $\left(* 10^{-3} \mathrm{~m} \mathrm{~s}^{-2}\right)$

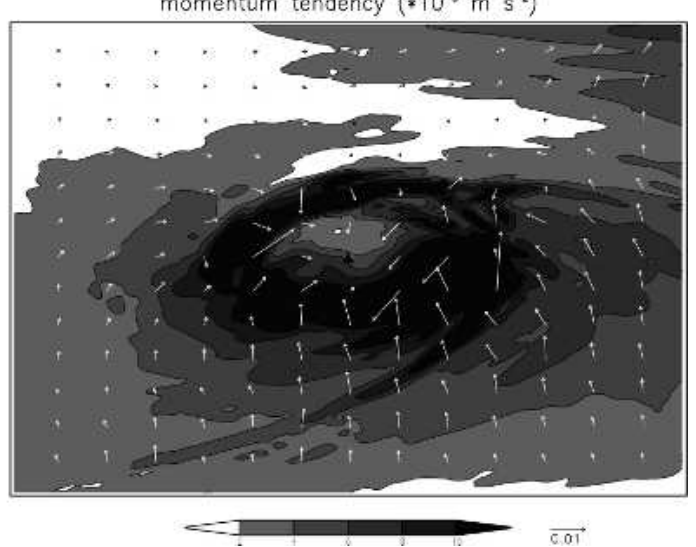

FIG. 9. (Continued)

to cold in its inner core. Such environmental gradients become superposed upon the transitioning TC, hypothesized to lead to the development of conveyor belts (Fig. 8a), and are amplified by the contrast between the developing cold thermal structure and warm air transported by these conveyor belts. The net result is an acceleration of the outer-core wind field-rather than just RMW and intensity changes that may be expected with the thermal evolution of a purely tropical TCand the right-of-track asymmetry noted in this expansion. Similar principles are considered toward understanding the inner-core wind field evolution, described next.

\section{c. Inner-core evolution}

The inner-core evolution of the transitioning cyclone's wind field is marked by the outward movement of the RMW noted during ET, occurring inside an approximately $200-\mathrm{km}$ radius. With intensifying tropical cyclones, the RMW contracts as the storm intensifies because of concentrated heating maximized inside the RMW resulting from convective- and subsidence-based heating processes (Shapiro and Willoughby 1982; Willoughby 1990). Conversely, the ET process is characterized by a transition from a warm-core to a cold-core vortex, suggesting cooling within the inner core of the cyclone. Following this, the methods of Shapiro and Willoughby (1982) and Willoughby (1990) are applied to the extratropical transition case to glean insight into the opposite evolution from a developing TC, that of the outward movement of the RMW. We transition from the isentropic to the isobaric vertical coordinate for simplicity and focus on the culmination of the ET process as that is when the greatest thermal evolution at low levels occurs.

Figure 10 depicts the azimuthally averaged temperature tendency at the $925-\mathrm{hPa}$ level at 1000 UTC 30 August 1998, near the completion of ET. Cooling is found within the inner core of the cyclone at this time as well as for several hours on either side of this time (not shown). Owing to the transition to a coldcore vortex, this is an expected result. Note that this evolution is also found in the vertical mean and is consistent with a rising mean sea level pressure. Figure 11 


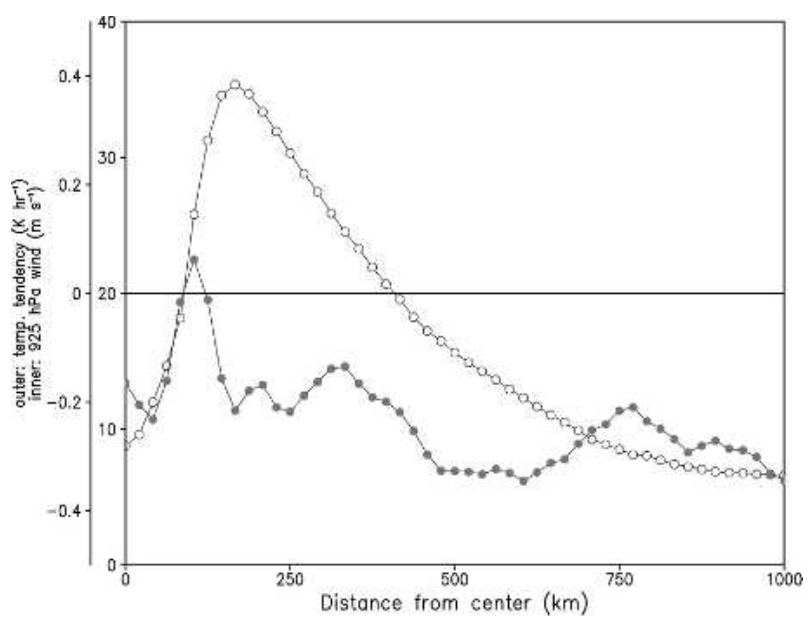

FIG. 10. The 925-hPa azimuthally averaged radial temperature tendency $\left(\mathrm{K} \mathrm{h}^{-1}\right.$; filled dots) and 925 -hPa azimuthally averaged tangential wind field ( $\mathrm{m} \mathrm{s}^{-1}$; open dots) at 1000 UTC 30 Aug 1998.

depicts the azimuthally averaged temperature tendency anomaly from the radial mean in the 950-200-hPa layer at 1000 UTC 30 August 1998. Integrated through the vertical, the inner core (e.g., inside the RMW at about $200-\mathrm{km}$ radius) cools at a faster rate than the outer core (e.g., outside the RMW at about $200-\mathrm{km}$ radius), suggesting a net weakening of the radial temperature gradient about the RMW associated with the TC undergoing cold-core ET. The oscillatory pattern noted is speculated to be a function of developing frontal circulations that are maintained through the azimuthally averaged fields.

The impact of this temperature evolution within the

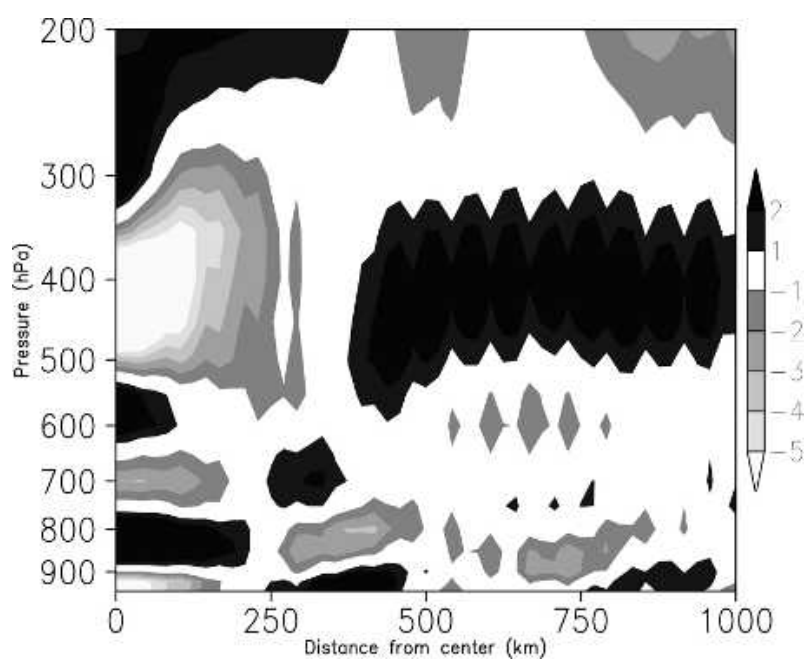

FIG. 11. Azimuthally averaged temperature tendency anomaly from the radial mean $\left(10^{-5} \mathrm{~K} \mathrm{~s}^{-1}\right)$ in the $950-200-\mathrm{hPa}$ layer at 1000 UTC 30 Aug 1998.
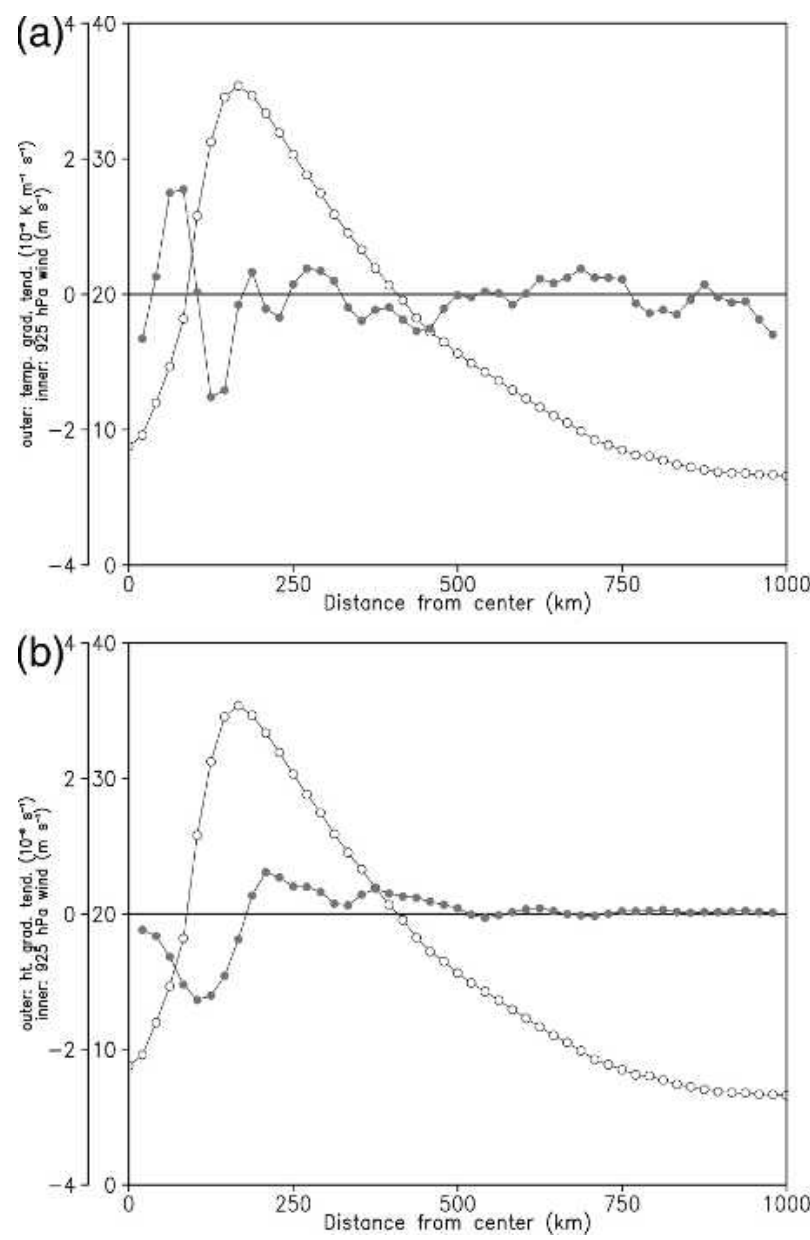

FIG. 12. (a) The 925-hPa azimuthally averaged time tendency of the radial temperature gradient $\left(10^{-9} \mathrm{~K} \mathrm{~m}^{-1} \mathrm{~s}^{-1}\right.$; filled dots $)$ and 925-hPa azimuthally averaged tangential wind field ( $\mathrm{m} \mathrm{s}^{-1}$; open dots) at 1000 UTC 30 Aug 1998; (b) as in (a), but for the 925-hPa azimuthally averaged time tendency of the radial isobaric height gradient $\left(10^{-8} \mathrm{~s}^{-1}\right.$; filled dots $)$

inner core upon the azimuthally averaged radial temperature and height gradients is examined next. Figure 12a depicts the evolution of the radial temperature gradient at 1000 UTC 30 August 1998. Coincident with the inner-core cooling, a weakening of the radial temperature gradient near and inside the RMW concordant with slight strengthening of the radial temperature gradient outside the RMW is noted (as expected from the time evolution of the streamfunction component to the isentropic momentum equation; cf. Figs. 9a,b). Thus, the effect of the inner-core cooling is to weaken the temperature gradients associated with the warm-core vortex. Figure $12 \mathrm{~b}$ depicts the evolution of the radial height gradient at 1000 UTC 30 August 1998. Weakening inside the RMW and slight tightening outside the RMW of the radial height gradient is noted and is a 
result that again is consistent through time, particularly for several hours prior to the analysis time. Hydrostatically, the weakening of the radial temperature gradient brings about this weakening of the radial height gradient. Ultimately, atmospheric and mass field adjustments occur because of the changes in the temperature field to attempt to maintain and restore hydrostatic balance.

From gradient wind balance, a weakening of the radial height gradient inside the RMW and a strengthening of the radial height gradient outside the RMW leads to an outward reorientation of the RMW, as observed here. The strengthening of the radial height gradients outside the RMW also leads to the acceleration of the wind field in the outer core (e.g., Fig. 12b), confirming the analyses and results presented in section $3 \mathrm{~b}$. With inner-core cooling driving this evolution, the outward movement of the RMW associated with ET is thus a natural outgrowth of the transition into a cold-core vortex. Independent of the cause of the cooling, the net effect is akin to a "reverse Sawyer-Eliassen" (Eliassen 1951) response and is qualitatively similar (yet opposite) to the contracting eyewall intensification cases of Shapiro and Willoughby (1982) and Willoughby (1990). This evolution can be further elucidated using a secondary circulation model, as discussed in the following section.

\section{Secondary circulation evolution}

\section{a. Model formulation}

The work of Hart et al. (2006) hypothesizes that the transition to a cold-core vortex during ET may be in response to adiabatic cooling not overcome by surface heat and moisture fluxes that is associated with an enhancement to the transitioning cyclone's secondary circulation by environmental forcing, particularly via momentum fluxes. The findings in section $3 \mathrm{c}$ argue that the thermal evolution of the circulation, particularly as it relates to the inner core cooling noted during ET, is integral to the outward movement of the RMW. Thus, to understand the environmental influences bringing about the inner-core outward movement of the RMW-the so-called reverse Sawyer-Eliassen response-plus gain further insight into the ET process as a whole, a baroclinic nonlinear secondary circulation diagnostic model is applied. This model is based upon the formulation of Montgomery et al. (2006, their section 7) and solves the elliptic PDE given by

$$
\begin{gathered}
\frac{\partial}{\partial r}\left(\frac{N^{2}}{r} \frac{\partial \psi}{\partial r}-\frac{\bar{\xi}}{r} \frac{\partial \bar{v}}{\partial z} \frac{\partial \psi}{\partial z}\right)+\frac{\partial}{\partial z}\left(-\frac{\bar{\xi}}{r} \frac{\partial \bar{v}}{\partial z} \frac{\partial \psi}{\partial r}+\frac{\bar{\xi} \bar{\eta}}{r} \frac{\partial \psi}{\partial z}\right) \\
=\frac{\partial \bar{Q}}{\partial r}-\frac{\partial}{\partial z}(\overline{\xi F}),
\end{gathered}
$$

where symbols used in (3) are as defined in Montgomery et al. (2006). In particular, $\bar{\xi}$ denotes the azimuthally averaged centrifugal stability, $\bar{F}$ denotes the momentum flux forcing, and $\bar{Q}$ denotes the heat flux forcing. This PDE gives a solution for the streamfunction $\bar{\psi}$ from which the mean radial and vertical motion fields $\bar{u}$ and $\bar{w}$ are calculated. The model is formulated using the pseudoheight vertical coordinate of Hoskins and Bretherton (1972) and extends between 0 and $10 \mathrm{~km}$ in the vertical and 0 and $1500 \mathrm{~km}$ in the radial directions $(\Delta z=500 \mathrm{~m}, \Delta r=15 \mathrm{~km})$. Momentum and heat flux forcing used to force the model comprise radial and vertical eddy vorticity and eddy heat fluxes, respectively, with an additional diabatic heating term computed as a residual in the heat flux formulation. These values are directly computed from the 12-km MM5 simulation data, as is the initial radial mean vorticity profile. Analyses are carried out at selected times during the transition life cycle of TC Bonnie with particular attention focused upon the times near and immediately preceding the completion of ET.

\section{b. Results}

Figure 13 depicts the flux-induced radial and vertical motion fields at 1000 UTC 30 August 1998. The momentum and heat flux forcings used to diagnose these fields are presented in Fig. 14. The primary secondary circulation is located in the lowest $5-6 \mathrm{~km}$ in altitude and within $500-\mathrm{km}$ radius. Note that at earlier times near the start of ET, the induced secondary circulation is of both greater vertical and horizontal extent (Fig. 15). This occurs as environmental forcing (not shown) acts to erode the initially upright and far-reaching secondary circulation found during TC Bonnie's tropical phase. The secondary circulation of the cyclone is oriented such that the strongest rising motion is found along and just outside the RMW (cf. Figs 13b and 15b to the azimuthally averaged wind field in Fig. 12a), gradually moving outward through time.

At this time-and throughout all simulation times during the transition of TC Bonnie-the momentum flux forcing is found to dominate over the heat flux forcing, a result consistent with the cold-core composite of Hart et al. (2006) with respect to the Eliassen-Palm flux formulation used in their work. Upon a breakdown of the components of this momentum flux forcing, it is found to be primarily driven by eddy vorticity fluxes about the RMW associated with its radial vorticity gradient (not shown). Specifically, the momentum flux forcing induces radial flow above and below the forcing, consistent with previous studies (Shapiro and Willoughby 1982; Molinari et al. 1995), which leads to rising motion within the column at the RMW. This re- 
(a)

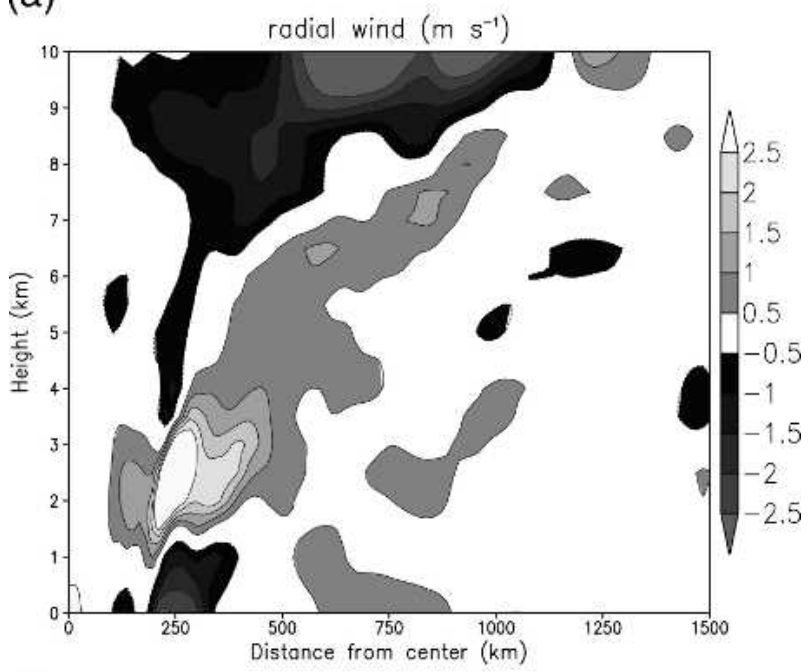

(b)

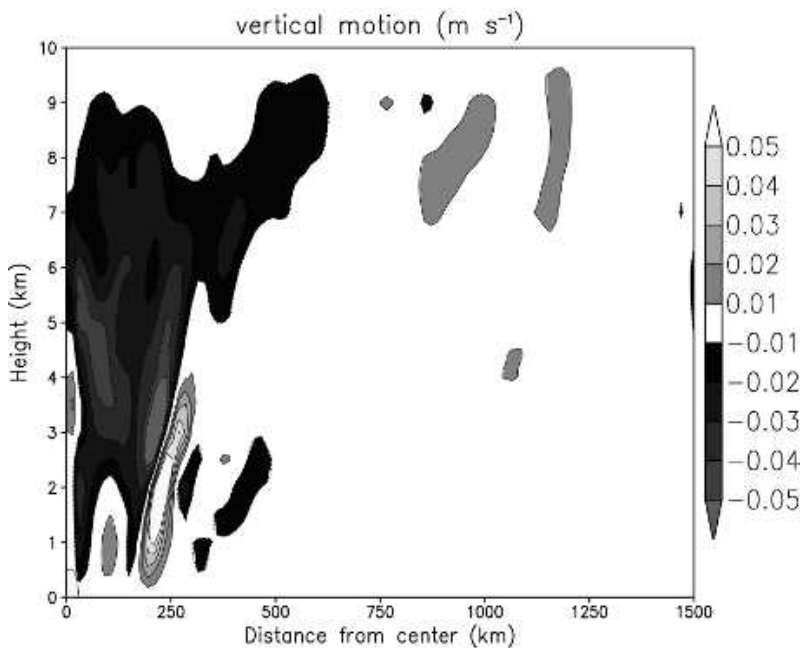

FIG. 13. (a) Radial (positive outward) and (b) vertical (positive upward) motion fields $\left(\mathrm{m} \mathrm{s}^{-1}\right)$ diagnosed at 1000 UTC 30 Aug 1998 between 0 and $10 \mathrm{~km}$ in altitude and 0 and $1500 \mathrm{~km}$ in radial distance.

sponse occurs in order to maintain thermal wind balance within the cyclone's environment in the presence of the momentum flux forcing (Molinari et al. 1995) and is hypothesized to bring about adiabatic cooling (Molinari et al. 1995; Hart et al. 2006) about the RMW. Weak compensating subsiding motion both inward and outward of the RMW and momentum flux forcing leads to compressional (adiabatic) warming in these regions, in part explaining the slight warming near the immediate center of the cyclone (e.g., Fig. 12a) and the tightening of the radial height and temperature gradients outside the RMW (e.g., Fig. 12b). The result is akin to a reverse Sawyer-Eliassen (Eliassen 1951) (a)

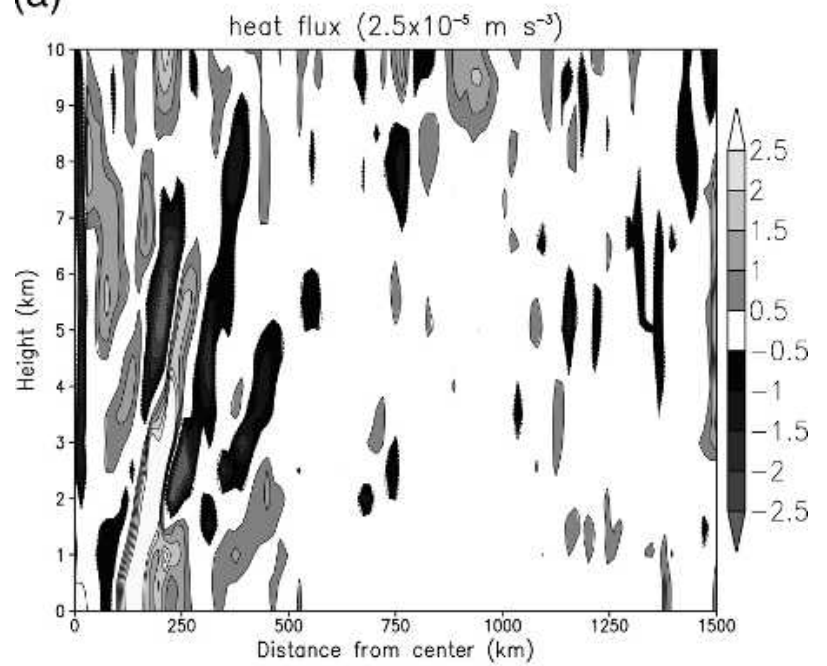

(b)

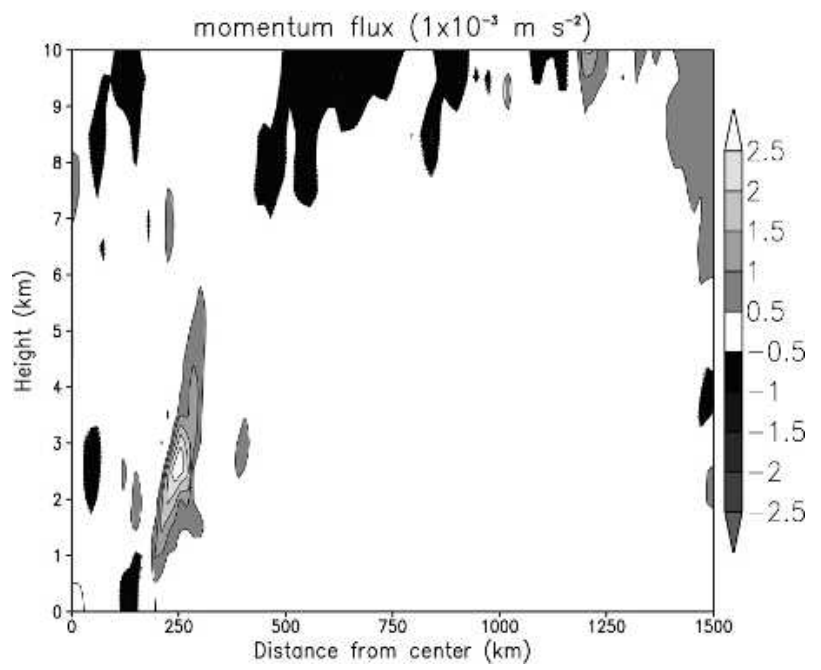

FIG. 14. (a) Heat $\left(2.5 \times 10^{-5} \mathrm{~m} \mathrm{~s}^{-3}\right)$ and (b) momentum $\left(10^{-3}\right.$ $\mathrm{m} \mathrm{s}^{-2}$ ) flux forcing as diagnosed from MM5 simulation data at 1000 UTC 30 Aug 1998 between 0 and $10 \mathrm{~km}$ in altitude and 0 and $1500 \mathrm{~km}$ in radial distance.

evolution, caused by the aforementioned cooling and heating patterns leading to the outward movement of the tightest radial temperature-and by extension, height-gradients. This results in changes to the location of the RMW (e.g., Fig. 5) and to the regions of maximum horizontal convergence at low levels (not shown), leading to the outward relocation of the rising branch of the cyclone's secondary circulation. The secondary circulation becomes more limited in vertical and radial extent during a cold-core weakening ET event due to changes in the inertial and static stabilities of the environment, two of the three factors governing the secondary circulation as described by Eliassen (1951). 
(a)

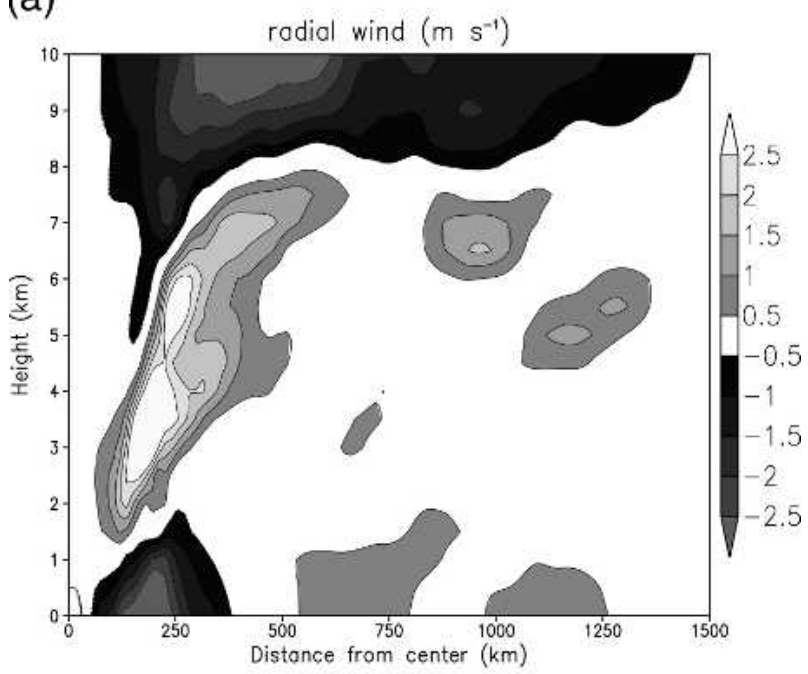

(b)

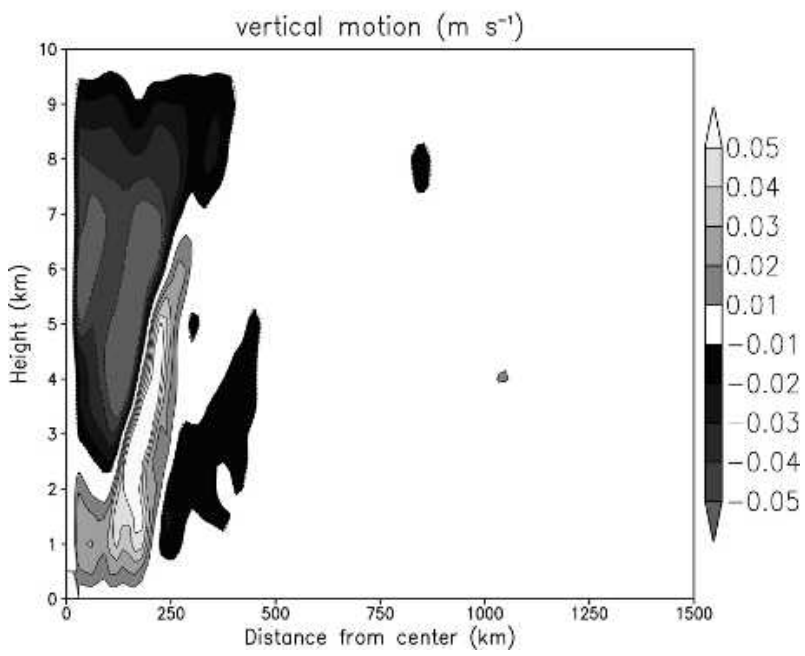

FIG. 15. As in Fig. 14, but at 2300 UTC 29 Aug 1998.

Despite this, the results from the diagnostic secondary circulation model suggest that at least at low levels, the secondary circulation response to the environment during ET is a similar yet opposite one to that of eyewall contraction, leading to the outward (rather than inward) movement of the RMW.

Aloft, the outward movement of the RMW occurs as the cyclone's secondary circulation decays from the top down in response to an unfavorable baroclinic environment, consistent with the ultimate decay of the cyclone. The greatest acceleration of the RMW aloft occurs prior to that at low levels, akin to that shown in Fig. 6, as a result of the erosion of the TC circulation and greater influence of the external environment at higher altitudes. This is highlighted by the limited vertical extent to the secondary circulation captured in Fig. 13 compared with Fig. 15. Note that this suggests that as the remnant cyclone continues to decay at higher altitudes during and after ET, other environmental processes (e.g., baroclinic energetics) play a dominant role in determining the ultimate evolution of the former TC and its wind field.

\section{Conclusions}

The wind field expansion associated with extratropically transitioning TCs can be decomposed into two concurrent evolutions involving both inner-core (inside the RMW) and outer-core (outside the RMW) components driving the outward movement of the RMW and acceleration of the outer wind field, respectively. With TC Bonnie (1998), the overall wind field expansion process occurs first at upper-tropospheric levels and progresses through time to lower levels, similar to the manner in which the inner-core cooling during a coldcore ET event is brought about during the ET process. Acceleration of the outer wind field occurs due to the net import of absolute angular momentum along inflowing trajectories within the cyclone's environment. Asymmetric compensating evolutions occur within the height and temperature fields of the cyclone's outer core as the cyclone's overall thermal structure changes, leading to the observed asymmetries in the outer wind field. Outward movement of the radial wind maximum occurs due to a secondary circulation response to relative cooling inside the cyclone's RMW. The overall circulation of the cyclone changes from relatively symmetric during its tropical phase to relatively asymmetric as this evolution occurs, with the greatest outer wind field acceleration noted where the developing asymmetric airstreams converge to the right of the cyclone's track.

Each evolution is a natural outgrowth of the ET process as a whole. Regarding the outer-core evolution, the ET process brings about the development of circulation flow asymmetries about the initially symmetric (or nearly so) cyclone in the form of conveyor belts (e.g., Browning 1999). These conveyor belts provide the mechanisms by which relatively warm air is transported into the outer envelope of the cyclone's circulation, particularly to the right of the cyclone's motion, as confirmed by trajectory analyses (Fig. 8a) and advection fields (Fig. 8b). Concurrently, the weakening of the TC's warm core and evolution to a cold-core structure results in a significant acceleration of the lower-level wind fields to the right of the cyclone's track and in the forward right quadrant of the cyclone (e.g., Figs. $7 \mathrm{~b}, \mathrm{~d}, \mathrm{f})$. Weaker outer-core wind field accelerations are noted in all other quadrants in response to the weaker development of height and temperature gradients away 
from the greatest external warm air transport. With respect to the inner-core evolution, the ET process is directly characterized by the transition from an initially warm-core tropical cyclone to a cold-core extratropical cyclone. The net cooling within the inner core, both integrated with height and with respect to the outer environment, is a marker of this transition and is argued to be a response to adiabatic cooling associated with rising motion forced by momentum fluxes about the RMW. This adiabatic cooling occurs with both tropical and extratropically transitioning cyclones, but is overcome in the former by surface heat fluxes over sufficiently warm SSTs (e.g., Emanuel 1986). As the RMW moves outward, the radial vorticity profile flattens at outer radii (not shown), combining with the previously described outer-core thermal evolution to bring about the outer wind field acceleration in all quadrants. Together, the end result is the outward movement of the RMW and flattening of the radially averaged tangential wind profile.

Changes in the orientation of material surfaces-and, by extension, in the nature of the conserved quantities-within the transitioning cyclone are implied with respect to the wind field evolution. During the tropical phase, surfaces of potential temperature (or more appropriately equivalent potential temperature) and momentum are parallel and are largely vertically oriented (Rotunno and Emanuel 1987). As the TC moves into the baroclinic environment of the midlatitudes, characterized by strong horizontal temperature gradients and large vertical wind shears, these surfaces no longer parallel. This evolution occurs as these temperature gradients are imposed across the transitioning TC, hypothesized to bring about a horizontal reorientation of the potential temperature contours, particularly at outer radii. Further implications from this reorientation of potential temperature and momentum contours during ET suggest that the evolution of material conservation (e.g., from conserving momentum to conserving potential temperature or a similar thermodynamic variable) may be important in understanding the full dynamics of the ET process. Future work will try to understand the nature of conserved quantities and flows with both extratropical and tropical cyclones and how they potentially change from one to the other during ET.

Other posttransition evolutions than that which TC Bonnie followed are possible, as highlighted by Hart et al. (2006). These include a transition into a warm seclusion (Shapiro and Keyser 1990) cyclone plus a distinction between cyclones that reintensify and those that only decay after transition. Each potential posttransition evolution results from a unique synoptic and TC environment, with differences between each evolu- tion largely focusing upon the location, magnitude, and orientation of the transition-inducing feature(s) (Hart et al. 2006). As a consequence, each posttransition evolution has a unique impact upon the evolution of the posttransition cyclone's wind field. The results during the ET process may be extended in an attempt to understand these posttransition evolutions. As an example, for warm seclusion cyclones, as the inner core warms with respect to the outer environment and radial temperature gradients tighten within the inner core, a recontraction of the radial wind maximum may be realized, consistent with the "tropical mode" (McTaggart-Cowan et al. 2004; Maue 2004) of development these cyclones often experience. A comprehensive study into these posttransition wind field evolutions may provide further insight into both the evolution of the cyclone wind field as well as the evolution of balance and conserved quantities for all cyclones.

Acknowledgments. Both authors were partially supported by Risk Prediction Initiative Grant RPI 04-2001. The first author was also partially supported by a Graduate Research Fellowship from the American Meteorological Society and the Office of Naval Research. The second author was also partially supported by an FSU FYAP grant. The framework for the secondary circulation model was graciously provided by Paul Reasor (The Florida State University) and suggested by Lance Bosart (University at Albany, State University of New York). The authors thank Lance Bosart, Phil Cunningham (The Florida State University), and T. N. Krishnamurti (The Florida State University) for their constructive comments and suggestions during the course of this work. The authors also thank Chris Fogarty (Environment Canada) and two anonymous reviewers for their comments and suggestions. All figures were created using the GrADS software package, and trajectory analyses were computed using the Vis $5 \mathrm{~d}$ software package.

\section{REFERENCES}

Avila, L. A., 1998: Hurricane Bonnie, 19-30 August 1998: NCEP preliminary report. [Available online at http://www.nhc. noaa.gov/1998bonnie.html.]

Blackadar, A. K., 1979: High resolution models of the planetary boundary layer. Advances in Environmental Science and Engineering, Vol. 1, J. Pfafflin and E. Ziegler, Eds., Gordon and Breach, 50-85.

Bowyer, P. J., 2000: Phenomenal waves with a transitioning tropical cyclone (Luis, the Queen, and the buoys). Preprints, 24th Conf. on Hurricanes and Tropical Meteorology, Fort Lauderdale, FL, Amer. Meteor. Soc., 294-295.

Browning, K. A., 1999: Mesoscale aspects of extratropical cyclones: An observational perspective. The Life Cycles of Ex- 
tratropical Cyclones, M. A. Shapiro and S. Grønås, Eds., Amer. Meteor. Soc., 265-283.

Dudhia, J., 1993: A non-hydrostatic version of the Penn StateNCAR Mesoscale Model: Validation tests and simulations of an Atlantic cyclone and cold front. Mon. Wea. Rev., 121, 1493-1513.

Eliassen, A., 1951: Slow thermally or frictionally controlled meridional circulation in a circular vortex. Astrophys. Norv., 5, $19-60$.

Emanuel, K. A., 1986: An air-sea interaction theory for tropical cyclones. Part I: Steady-state maintenance. J. Atmos. Sci., 43, 585-604.

Evans, A. C., 2006: Dynamics of the wind field expansion associated with extratropically transitioning tropical cyclones. M.S. thesis, Dept. of Meteorology, The Florida State University, $111 \mathrm{pp}$.

Evans, J. L., and R. Hart, 2003: Objective indicators of the life cycle evolution of extratropical transition for Atlantic tropical cyclones. Mon. Wea. Rev., 131, 909-925.

_ - J. M. Arnott, and F. Chiaromonte, 2006: Evaluation of operational model cyclone structure forecasts during extratropical transition. Mon. Wea. Rev., 134, 3054-3072.

Harr, P. A., and R. L. Elsberry, 2000: Extratropical transition of tropical cyclones over the western North Pacific. Part I: Evolution of structural characteristics during the transition process. Mon. Wea. Rev., 128, 2613-2633.

Hart, R. E., 2003: A cyclone phase space derived from thermal wind and thermal asymmetry. Mon. Wea. Rev., 131, 585-616.

_ J. J. L. Evans, and C. Evans, 2006: Synoptic composites of the extratropical transition life cycle of North Atlantic tropical cyclones: Factors determining posttransition evolution. Mon. Wea. Rev., 134, 553-578.

Hoskins, B. J., and F. P. Bretherton, 1972: Atmospheric frontogenesis models: Mathematical formulation and solution. $J$. Atmos. Sci., 29, 11-37.

Jones, S. C., and Coauthors, 2003: The extratropical transition of tropical cyclones: Forecast challenges, current understanding, and future directions. Wea. Forecasting, 18, 1052-1092.

Kain, J. S., and J. M. Fritsch, 2003: The Kain-Fritsch scheme. The Representation of Cumulus Convection in Numerical Models. Meteor. Monogr., No. 24, Amer. Meteor. Soc., 165-170.

Klein, P. M., P. A. Harr, and R. L. Elsberry, 2000: Extratropical transition of northwest Pacific tropical cyclones: An overview and conceptual model of the transformation stage. Wea. Forecasting, 15, 373-395.

Maue, R. N., 2004: Evolution of frontal structure associated with extratropically transitioning hurricanes. M.S. thesis, Dept. of Meteorology, The Florida State University, 109 pp.
McBride, J. L., 1981a: Observational analysis of tropical cyclone formation. Part I: Basic description of datasets. J. Atmos. Sci., 38, 1117-1131.

_ 1981b: Observational analysis of tropical cyclone formation. Part III: Budget analysis. J. Atmos. Sci., 38, 1152-1166.

McTaggart-Cowan, R., J. R. Gyakum, and M. K. Yau, 2004: The impacts of tropical remnants on extratropical cyclogenesis: Case study of hurricanes Danielle and Earl (1998). Mon. Wea. Rev., 132, 1933-1951.

Molinari, J., S. Skubis, and D. Vollaro, 1995: External influences on hurricane intensity. Part III: Potential vorticity structure. J. Atmos. Sci., 52, 3593-3606.

Montgomery, M. T., M. E. Nicholls, T. A. Cram, and A. B. Saunders, 2006: A vortical hot tower route to tropical cyclogenesis. J. Atmos. Sci., 63, 355-386.

National Hurricane Center, cited 2006: National Hurricane Center forecast verification. [Available online at http://www.nhc. noaa.gov/verification/verify4.shtml.]

— - cited 2007: Hurricane Bonnie advisory archive. [Available online at http://www.nhc.noaa.gov/1998BONNIEadv.html.]

Powell, M. D., S. H. Houston, L. R. Amat, and N. MorisseauLeroy, 1998: The HRD real-time hurricane wind analysis system. J. Wind Eng. Indust. Aerodyn., 77, 53-64.

Reisner, J., R. M. Rasmussen, and R. T. Bruintjes, 1998: Explicit forecasting of supercooled liquid water in winter storms using the MM5 mesoscale model. Quart J. Roy. Meteor. Soc., 124, 1071-1107.

Ritchie, E. A., and R. L. Elsberry, 2001: Simulations of the transformation stage of the extratropical transition of tropical cyclones. Mon. Wea. Rev., 129, 1462-1480.

Rotunno, R., and K. A. Emanuel, 1987: An air-sea interaction theory for tropical cyclones. Part II: Evolutionary study using a nonhydrostatic axisymmetric numerical model. J. Atmos. Sci., 44, 542-561.

Shapiro, L. J., and H. E. Willoughby, 1982: The response of balanced hurricanes to local sources of heat and momentum. $J$. Atmos. Sci., 39, 378-394.

Shapiro, M. A., 1975: Simulation of upper-level frontogenesis with a 20-level isentropic coordinate primitive equation model. Mon. Wea. Rev., 103, 591-604.

—, and D. Keyser, 1990: Fronts, jet streams, and the tropopause. Extratropical Cyclones: The Erik Palmén Memorial Volume, C. W. Newton and E. O. Holopainen, Eds., Amer. Meteor. Soc., 167-191.

Willoughby, H. E., 1990: Temporal changes of the primary circulation in tropical cyclones. J. Atmos. Sci., 47, 242-264. 